\title{
Construction of singular limits for a strongly perturbed four-dimensional Navier problem with exponentially dominated nonlinearity and nonlinear terms
}

\section{Sami Baraket ${ }^{1}$, Souhail Chebbi ${ }^{2 *}$ and Nejmeddine Chorfi ${ }^{2}$}

\section{"Correspondence:}

schebbi@ksu.edu.sa

${ }^{2}$ Department of Mathematics,

College of Science, King Saud

University, Riyadh, Saudi Arabia

Full list of author information is

available at the end of the article

\begin{abstract}
Given a bounded open regular set $\Omega \in \mathbb{R}^{4}, x_{1}, x_{2}, \ldots, x_{m} \in \Omega, \lambda, \rho>0, \gamma \in(0,1)$, and $\mathscr{Q}_{\lambda}$ some nonlinear operator (which will be defined later), we prove that the problem

$$
\Delta^{2} u+\mathscr{Q}_{\lambda}(u)=\rho^{4}\left(e^{u}+e^{\gamma u}\right)
$$

has a positive weak solution in $\Omega$ with $u=\Delta u=0$ on $\partial \Omega$, which is singular at each $x_{i}$ as the parameters $\lambda$ and $\rho$ tend to 0 .
\end{abstract}

Keywords: Biharmonic operator; Nonlinear operator; Singular limits; Green's function; Nonlinear domain decomposition method

\section{Introduction and statement of results}

Semilinear equations involving fourth order elliptic operator and exponential nonlinearity appear naturally in conformal geometry and in particular in the prescription of the socalled Q-curvature in four-dimensional Riemannian manifolds $[9,10]$

$$
Q_{g}=\frac{1}{12}\left(-\Delta_{g} S_{g}+S_{g}^{2}-3\left|\operatorname{Ric}_{g}\right|^{2}\right)
$$

where $\operatorname{Ric}_{g}$ denotes the Ricci tensor and $S_{g}$ is the scalar curvature of the metric $g$. Recall that the $Q$-curvature changes under a conformal change of metric

$$
g_{w}=e^{2 w} g,
$$

according to

$$
P_{g} w+2 Q_{g}=2 \tilde{Q}_{g_{w}} e^{4 w}
$$

where

$$
P_{g}:=\Delta_{g}^{2}+\delta\left(\frac{2}{3} S_{g} I-2 \mathrm{Ric}_{g}\right) d
$$

(c) The Author(s) 2019. This article is distributed under the terms of the Creative Commons Attribution 4.0 International License (http://creativecommons.org/licenses/by/4.0/), which permits unrestricted use, distribution, and reproduction in any medium, provided you give appropriate credit to the original author(s) and the source, provide a link to the Creative Commons license, and indicate if changes were made. 
is the Paneitz operator, which is an elliptic fourth order partial differential operator [10] and which transforms according to

$$
e^{4 w} P_{e^{2 w} g}=P_{g}
$$

under a conformal change of metric $g_{w}:=e^{2 w} g$. In the special case where the manifold is the Euclidean space, the Paneitz operator is simply given by

$$
P_{g_{\text {eucl }}}=\Delta^{2},
$$

in which case (1) can be written as

$$
\Delta^{2} w=\tilde{Q} e^{4 w}
$$

the solutions of which give rise to the conformal metric $g_{w}=e^{2 w} g_{\text {eucl }}$ whose $Q$-curvature is given by $\tilde{Q}$. There is by now extensive literature about this problem, and we refer to [10] and [15] for references and recent developments.

In this paper, we are interested in positive solutions of

$$
\begin{cases}\Delta^{2} u+\mathscr{Q}_{\lambda}(u)=\rho^{4}\left(e^{u}+e^{\gamma u}\right) & \text { in } \Omega, \\ u=\Delta u=0 & \text { on } \partial \Omega\end{cases}
$$

with a regular bounded open domain $\Omega \subset \mathbb{R}^{4}, \lambda, \rho>0, \gamma \in(0,1)$ and a nonlinear operator $\mathscr{Q}_{\lambda}$ given by

$$
\begin{aligned}
\mathscr{Q}_{\lambda}(u):= & \lambda\left[(\Delta u)^{2}+\Delta\left(|\nabla u|^{2}\right)+2 \nabla u \cdot \nabla(\Delta u)\right] \\
& +2 \lambda^{2}\left[\Delta u|\nabla u|^{2}+\nabla u \cdot \nabla\left(|\nabla u|^{2}\right)\right]+\lambda^{3}|\nabla u|^{4} .
\end{aligned}
$$

Using the following transformation:

$$
w:=\left(\lambda \rho^{4} e^{u}\right)^{\lambda}
$$

so, if $u$ is a solution of (2), then $w$ solves the following equation:

$$
\Delta^{2} w=w^{\frac{\lambda+1}{\lambda}}+\theta w^{\frac{\gamma+\lambda}{\lambda}} \text { in } \Omega
$$

where $\theta=\left(\lambda \rho^{4}\right)^{1-\gamma}$. Remark that the exponent $q=\frac{\lambda+1}{\lambda}$ tends to $\infty$ as $\lambda$ tends to 0 .

We denote by $\varepsilon$ the smallest positive parameter satisfying

$$
\rho^{4}=\frac{384 \varepsilon^{4}}{\left(1+\varepsilon^{2}\right)^{4}}
$$

Remark that $\rho \sim \varepsilon$ as $\varepsilon \longrightarrow 0$. We will suppose in the following that

$$
\left(A_{\lambda}\right): \quad \lambda^{1+\delta / 2} \varepsilon^{-\delta}=\mathcal{O}(1) \quad \text { as } \varepsilon \rightarrow 0 \text { for any } \delta \in(0,1) .
$$


In particular, if we take $\lambda=\mathcal{O}\left(\varepsilon^{2 / 3}\right)$, then condition $\left(A_{\lambda}\right)$ is satisfied. Under assumption $\left(A_{\lambda}\right)$, we can treat equation (2) as a perturbation of the following equation:

$$
\Delta^{2} u=\rho^{4}\left(e^{u}+e^{\gamma u}\right) \quad \text { in } \Omega \subset \mathbb{R}^{4} .
$$

Our question is as follows: Does there exist $u_{\varepsilon}$ a sequence of solutions which converges to some singular function as the parameters $\varepsilon$ and $\lambda$ tend to 0 ?

In [2], Baraket et al. gave a positive answer to the above question for the following problem:

$$
\begin{cases}\Delta^{2} u+\mathscr{Q}_{\lambda}(u)=\rho^{4} e^{u} & \text { in } \Omega \\ u=\Delta u=0 & \text { on } \partial \Omega\end{cases}
$$

with a regular bounded open domain $\Omega \subset \mathbb{R}^{4}$. They gave a sufficient condition for problem (7) to have a weak solution in $\Omega$ which is singular at some points $\left(x_{i}\right)_{1 \leq i \leq m}$ as $\rho$ and $\lambda$ are small parameters satisfying $\left(A_{\lambda}\right)$.

In case $\lambda=0$, the authors in [3] gave also a positive answer to the following problem:

$$
\begin{cases}\Delta^{2} u=\rho^{4}\left(e^{u}+e^{\gamma u}\right) & \text { in } \Omega \\ u=\Delta u=0 & \text { on } \partial \Omega\end{cases}
$$

for $\gamma \in(0,1)$ as $\rho$ tends to 0 . When $\lambda=0$ and $\gamma=0$, problem (2) reduces to

$$
\begin{cases}\Delta^{2} u=\rho^{4} e^{u} & \text { in } \Omega \\ u=\Delta u=0 & \text { on } \partial \Omega\end{cases}
$$

In 1996, Wei in [20] studied the behavior of solutions to the following nonlinear problem in $\mathbb{R}^{4}$. More precisely, consider the following problem:

$$
\begin{cases}\Delta^{2} u=\lambda f(u) & \text { in } \Omega, \\ u=\Delta u=0 & \text { on } \partial \Omega .\end{cases}
$$

Before showing his result, we will introduce some notations. Let $G\left(x, x^{\prime}\right)$ defined over $\Omega \times$ $\Omega$, the Green's function associated with the bi-Laplacian operator with a Navier boundary conditions, which is the solution of

$$
\begin{cases}\Delta_{x}^{2} G\left(x, x^{\prime}\right)=64 \pi^{2} \delta_{x=x^{\prime}} & \text { in } \Omega \\ G\left(x, x^{\prime}\right)=\Delta_{x} G\left(x, x^{\prime}\right)=0 & \text { on } \partial \Omega .\end{cases}
$$

and denote by $H\left(x, x^{\prime}\right)=G\left(x, x^{\prime}\right)+8 \log \left|x-x^{\prime}\right|$ its smooth part. Consider now the functional

$$
E:\left(x^{1}, \ldots, x^{m}\right) \in\left(\mathbb{R}^{4}\right)^{m} \longmapsto \sum_{j=1}^{m} H\left(x^{j}, x^{j}\right)+\sum_{j \neq l} G\left(x^{j}, x^{l}\right)
$$


Denote by $u^{*}$ the solution of

$$
\begin{cases}\Delta^{2} u^{*}=64 \pi^{2} \sum_{i=1}^{m} \delta_{x^{i}} & \text { in } \Omega, \\ u^{*}=\Delta u^{*}=0 & \text { on } \partial \Omega .\end{cases}
$$

The author proved the following result.

Theorem 1 ([20]) Let $\Omega$ be a smooth bounded domain in $\mathbb{R}^{4}$ and $f$ be a smooth nonnegative increasing function such that

$$
e^{-u} f(u) \text { and } e^{-u} \int_{0}^{u} f(s) d s \text { tends to } 1 \text {, as } u \longrightarrow+\infty
$$

For $u_{\lambda}$ solution of $(10)$, denote by $\Sigma_{\lambda}=\lambda \int_{\Omega} f\left(u_{\lambda}\right) d x$. Then three cases occur:

1. $\Sigma_{\lambda} \longrightarrow 0$, therefore $\left\|u_{\lambda}\right\|_{L^{\infty}(\Omega)} \longrightarrow 0$ as $\lambda \longrightarrow 0$.

2. $\Sigma_{\lambda} \longrightarrow+\infty$, then $u_{\lambda} \longrightarrow+\infty$ as $\lambda \longrightarrow 0$.

3. $\Sigma_{\lambda} \longrightarrow 64 \pi^{2} m$ for some positive integer $m$. Then the limiting function $u^{*}=\lim _{\lambda \rightarrow 0} u_{\lambda}$ has $m$ blow-up points $\left\{x^{1}, \ldots, x^{m}\right\}$, where $u_{\lambda}\left(x^{i}\right) \longrightarrow+\infty$ as $\lambda \longrightarrow 0$. Moreover, $\left(x^{1}, \ldots, x^{m}\right)$ is a critical point of $E$.

Recently, in [4], the authors constructed non-minimal solutions of problem (9) with singular limit as the parameter $\rho$ tends to 0 . Their results, which give the converse of case (3) given in the last theorem, can be stated as follows.

Theorem 2 ([4]) Let $\Omega$ be a smooth open subset of $\mathbb{R}^{4}$. Assume that $\left(x^{1}, \ldots, x^{m}\right)$ is a nondegenerate critical point of $E$, then there exist $\rho_{0}>0$ and a one-parameter family $\left(u_{\rho}\right)_{\rho \in\left(0, \rho_{0}\right)}$ of solutions of (9) such that

$$
\lim _{\rho \rightarrow 0} u_{\rho}=u^{*}, \quad \text { in } \mathcal{C}_{\text {loc }}^{4, \alpha}\left(\Omega-\left\{x^{1}, \ldots, x^{m}\right\}\right) .
$$

Our main result is the following.

Theorem 3 Given $\alpha \in(0,1)$. Let $\Omega$ be an open smooth bounded set of $\mathbb{R}^{4}, \lambda>0$ satisfying condition $\left(A_{\lambda}\right)$, and $S=\left\{x_{1}, \ldots, x_{m}\right\} \subset \Omega$ be a nonempty set. Assume that $\left(x_{1}, \ldots, x_{m}\right)$ is a nondegenerate critical point of the function

$$
\mathscr{F}\left(x_{1}, \ldots, x_{m}\right)=\sum_{j=1}^{m} H\left(x_{j}, x_{j}\right)+\sum_{i \neq j} G\left(x_{i}, x_{j}\right) \quad \text { in }(\Omega)^{m},
$$

then there exist $\rho_{0}>0, \lambda_{0}>0$, and a family $\left\{u_{\rho, \lambda}\right\}_{0<\rho<\rho_{0}, 0<\lambda<\lambda_{0}}$ of solutions of $(2)$ such that

$$
\lim _{\substack{\rho \rightarrow 0 \\ \lambda \rightarrow 0}} u_{\rho, \lambda}=\sum_{j=1}^{m} G\left(x_{j}, \cdot\right) \quad \text { in } \mathcal{C}_{\text {loc }}^{4, \alpha}\left(\Omega-\left\{x_{1}, \ldots, x_{m}\right\}\right) .
$$




\section{Construction of the approximate solution}

We first describe the rotationally symmetric approximate solutions of

$$
\Delta^{2} u-\rho^{4} e^{u}=0
$$

in $\mathbb{R}^{4}$, which will be crucial in the construction of the approximate solution. Given $\varepsilon>0$, we define

$$
u_{\varepsilon, \tau}(x):=4 \log \left(1+\varepsilon^{2}\right)+4 \log \tau-4 \log \left(\varepsilon^{2}+(\tau|x|)^{2}\right),
$$

which is clearly a solution of (14) when

$$
\rho^{4}=\frac{384 \varepsilon^{4}}{\left(1+\varepsilon^{2}\right)^{4}}
$$

For $\tau>0$, we remark that equation (14) is invariant under some dilation in the following sense: If $u$ is a solution of (14), then

$$
\tau \mapsto u(\tau \cdot)+4 \log \tau
$$

is also solution of (14). So, for $\varepsilon>0$ and $\tau>0$, we denote by $u_{\varepsilon, \tau}$ the element of this new family of radial solutions of (14).

For $\varepsilon=\tau=1$, we denote by $u_{1}=u_{1,1}$ this particular solution. We also define the following linear fourth order elliptic operator:

$$
\mathscr{L}:=\Delta^{2}-\frac{384}{\left(1+|x|^{2}\right)^{4}}
$$

which corresponds to the linearization of (14) about the solution $u_{1}$.

\subsection{Radial solution on $\mathbb{R}^{4}$}

For all $\varepsilon, \tau, \lambda>0, \delta \in(0,1)$, we set

$$
R_{\varepsilon, \lambda}:=\tau r_{\varepsilon, \lambda} / \varepsilon
$$

where

$$
r_{\varepsilon, \lambda}:=\max \left(\sqrt{\varepsilon}, \sqrt{\lambda}, \varepsilon^{4(1-\gamma)-\delta / 4}\right) .
$$

The classification of bounded solutions of $\mathbb{L} w=0$ in $\mathbb{R}^{4}$ is well known. Some solutions are easy to find. For example, we can define

$$
\phi_{0}(x):=r \partial_{r} u_{1}(x)+4=4 \frac{1-r^{2}}{1+r^{2}},
$$

where $r=|x|$. Clearly, $\mathbb{L} \phi_{0}=0$, and this reflects the fact that (14) is invariant under the group of dilations $\tau \mapsto u(\tau \cdot)+4 \log \tau$. We also define, for $i=1, \ldots, 4$,

$$
\phi_{i}(x):=-\partial_{x_{i}} u_{1}(x)=\frac{8 x_{i}}{1+|x|^{2}},
$$


which are also solutions of $\mathbb{L} \phi_{i}=0$ since these solutions correspond to the invariance of the equation under the group of translations $a \mapsto u(\cdot+a)$.

Then we have the following classification.

Lemma 1 ([4]) Any bounded solution of $\mathscr{L} w=0$ defined in $\mathbb{R}^{4}$ is a linear combination of $\phi_{i}$ for $i=0,1, \ldots, 4$.

Let $B_{r}$ denote the ball of radius $r$ centered at the origin in $\mathbb{R}^{4}$.

Definition 1 Given $k \in \mathbb{N}, \alpha \in(0,1)$ and $\mu \in \mathbb{R}$, we introduce the Hölder weighted spaces $\mathcal{C}_{\mu}^{k, \alpha}\left(\mathbb{R}^{4}\right)$ as the space of functions $w \in \mathcal{C}_{\text {loc }}^{k, \alpha}\left(\mathbb{R}^{4}\right)$ for which the following norm

$$
\|w\|_{\mathcal{C}_{\mu}^{k, \alpha}\left(\mathbb{R}^{4}\right)}:=\|w\|_{\mathcal{C}^{k, \alpha}\left(\bar{B}_{1}\right)}+\sup _{r \geq 1}\left(\left(1+r^{2}\right)^{-\delta / 2}\|w(r \cdot)\|_{\mathcal{C}_{\mu}^{k, \alpha}\left(\bar{B}_{1}-B_{1 / 2}\right)}\right)
$$

is finite.

We also define

$$
\mathcal{C}_{\mathrm{rad}, \mu}^{k, \alpha}\left(\mathbb{R}^{4}\right)=\left\{f \in \mathcal{C}_{\mu}^{k, \alpha}\left(\mathbb{R}^{4}\right) \text {; such that } f(x)=f(|x|), \forall x \in \mathbb{R}^{4}\right\} .
$$

As a consequence of the result of Lemma 1 , we recall the surjectivity result of $\mathscr{L}$.

\section{Proposition 1 ([4])}

1. Assume that $\mu>1$ and $\mu \notin \mathbb{Z}$, then

$$
\begin{aligned}
L_{\mu}: & \quad \mathcal{C}_{\mu}^{4, \alpha}\left(\mathbb{R}^{4}\right) \longrightarrow \mathcal{C}_{\mu-4}^{0, \alpha}\left(\mathbb{R}^{4}\right), \\
& w \longmapsto \mathscr{L} w
\end{aligned}
$$

is surjective.

2. Assume that $\delta>0$ and $\delta \notin \mathbb{Z}$, then

$$
\begin{aligned}
L_{\delta}: \quad & \left.\mathcal{C}_{\text {rad }, \delta}^{4, \alpha}\left(\mathbb{R}^{4}\right)\right) \longrightarrow \mathcal{C}_{\text {rad }, \delta-4}^{0, \alpha}\left(\mathbb{R}^{4}\right), \\
& w \longmapsto \mathscr{L} w
\end{aligned}
$$

is surjective.

We set $\bar{B}_{1}^{*}=\bar{B}_{1}-\{0\}$.

Definition 2 Given $k \in \mathbb{N}, \alpha \in(0,1)$, and $\mu \in \mathbb{R}$, we introduce the Hölder weighted spaces $\mathcal{C}_{\mu}^{k, \alpha}\left(\bar{B}_{1}^{*}\right)$ as the space of functions in $\mathcal{C}_{\text {loc }}^{k, \alpha}\left(\bar{B}_{1}^{*}\right)$ for which the following norm

$$
\|u\|_{\mathcal{C}_{\mu}^{k, \alpha}\left(\bar{B}_{1}^{*}\right)}=\sup _{r \leq 1 / 2}\left(r^{-\mu}\|u(r \cdot)\|_{\mathcal{C}^{k, \alpha}\left(\bar{B}_{2}-B_{1}\right)}\right)
$$

is finite. 
Then we define the subspace of radial functions in $\mathcal{C}_{\delta}^{k, \alpha}\left(\bar{B}_{1}^{*}\right)$ by

$$
\mathcal{C}_{\text {rad }, \delta}^{k, \alpha}\left(\bar{B}_{1}^{*}\right)=\left\{f \in \mathcal{C}_{\delta}^{k, \alpha}\left(\mathbb{R}^{4}\right) \text {; such that } f(x)=f(|x|), \forall x \in \bar{B}_{1}^{*}\right\} .
$$

Our aim now is the construction of a radial solution $u$ of

$$
\Delta^{2} u+\mathscr{Q}_{\lambda}(u)-\rho^{4}\left(e^{u}+e^{\gamma u}\right)=0 \quad \text { in } \bar{B}_{r_{\varepsilon, \lambda}} .
$$

Thanks to the following transformation

$$
v(x)=u\left(\frac{\varepsilon}{\tau} x\right)+8 \log \varepsilon-4 \log \left(\tau\left(1+\varepsilon^{2}\right) / 2\right),
$$

then Eq. (16) can be written as

$$
\Delta^{2} v+\mathscr{Q}_{\lambda}(v)-24\left(e^{\nu}+\frac{2^{4(1-\gamma)} \varepsilon^{8(1-\gamma)}}{\left(\left(1+\varepsilon^{2}\right) \tau\right)^{4(1-\gamma)}} e^{\gamma \nu}\right)=0 \quad \text { in } \bar{B}_{R_{\varepsilon, \lambda}} .
$$

Now, we look for a solution of (17) of the form

$$
v(x)=u_{1}(x)+h(x)
$$

this amounts to solving

$$
\begin{aligned}
\mathscr{L} h= & \frac{384}{\left(1+|x|^{2}\right)^{4}}\left(e^{h}-h-1\right)+\frac{384 \varepsilon^{8(1-\gamma)}}{\left(\left(1+\varepsilon^{2}\right) \tau\right)^{4(1-\gamma)}\left(1+|x|^{2}\right)^{4 \gamma}} e^{\gamma h} \\
& -\mathscr{Q}_{\lambda}\left(u_{1}+h\right)
\end{aligned}
$$

in $\bar{B}_{R_{\varepsilon, \lambda}}$. We will need the following definition.

Definition 3 Given $\bar{r} \geq 1, k \in \mathbb{N}, \alpha \in(0,1)$, and $\delta \in \mathbb{R}$, the weighted space $\mathcal{C}_{\delta}^{k, \alpha}\left(B_{\bar{r}}\right)$ is defined to be the space of functions $w \in \mathcal{C}^{k, \alpha}\left(B_{\bar{r}}\right)$ endowed with the norm

$$
\|w\|_{\mathcal{C}_{\delta}^{k, \alpha}\left(\bar{B}_{\bar{r}}\right)}:=\|w\|_{\mathcal{C}^{k, \alpha}\left(B_{1}\right)}+\sup _{1 \leq r \leq \bar{r}}\left(r^{-\delta}\|w(r \cdot)\|_{\mathcal{C}^{k, \alpha}\left(\bar{B}_{1}-B_{1 / 2}\right)}\right)
$$

For all $\sigma \geq 1$, we denote by

$$
\mathscr{E}_{\sigma}: \mathcal{C}_{\delta}^{0, \alpha}\left(\bar{B}_{\sigma}\right) \longrightarrow \mathcal{C}_{\delta}^{0, \alpha}\left(\mathbb{R}^{4}\right)
$$

the extension operator defined by

$$
\mathscr{E}_{\sigma}(f)(x)=\chi\left(\frac{|x|}{\sigma}\right) f\left(\sigma \frac{x}{|x|}\right)
$$

where $t \longmapsto \chi(t)$ is a smooth nonnegative cut-off function identically equal to 1 for $t \geq 2$ and identically equal to 0 for $t \leq 1$. It is easy to check that there exists a constant $c=c(\delta)>$ 0 , independent of $\sigma \geq 1$, such that

$$
\left\|\mathscr{E}_{\sigma}(w)\right\|_{\mathcal{C}_{\delta}^{0, \alpha}\left(\mathbb{R}^{4}\right)} \leq c\|w\|_{\mathcal{C}_{\delta}^{0, \alpha}\left(\bar{B}_{\sigma}\right)}
$$


We fix

$$
\delta \in(0,1)
$$

and denote by $\mathscr{G}_{\delta}$ a right inverse of $\mathscr{L}_{\delta}$ assured by Proposition 1 . Now, we use the result of Proposition 1 to rephrase the nonlinear equation (18) as a fixed point problem. Hence, to obtain a solution of (18), it is enough to find a fixed point $h$ in a small ball of $\mathcal{C}_{\text {rad, } \delta}^{4, \alpha}\left(\mathbb{R}^{4}\right)$ for the mapping

$$
h \mapsto \mathscr{N}(h):=\mathscr{G}_{\delta} \circ \mathscr{E}_{\delta} \circ \mathscr{R}(h),
$$

where

$$
\begin{aligned}
\mathscr{R}(h):= & \frac{384}{\left(1+|x|^{2}\right)^{4}}\left(e^{h}-h-1\right)+\frac{384 \varepsilon^{8(1-\gamma)}}{\left(\left(1+\varepsilon^{2}\right) \tau\right)^{4(1-\gamma)}\left(1+|x|^{2}\right)^{4 \gamma}} e^{\gamma h} \\
& -\mathscr{Q}_{\lambda}\left(u_{1}+h\right) .
\end{aligned}
$$

We have

$$
\begin{aligned}
\mathscr{R}(0)= & -\lambda\left[(\Delta u)^{2}+\Delta\left(|\nabla u|^{2}\right)+2 \nabla u \cdot \nabla(\Delta u)\right] \\
& -2 \lambda^{2}\left[\Delta u|\nabla u|^{2}+\nabla u \cdot \nabla\left(|\nabla u|^{2}\right)\right]-\lambda^{3}|\nabla u|^{4} .
\end{aligned}
$$

Recall that

$$
u_{1}=4 \log (2)-4 \log \left(1+r^{2}\right)
$$

then

$$
\left|\nabla u_{1}\right|^{2}=64 \frac{r^{2}}{\left(1+r^{2}\right)^{2}}, \quad \Delta u_{1}=-16 \frac{2+r^{2}}{\left(1+r^{2}\right)^{2}} \quad \text { and } \quad \Delta\left(\left|\nabla u_{1}\right|^{2}\right)=512 \frac{1-2 r^{2}}{\left(1+r^{2}\right)^{4}}
$$

Hence,

$$
\begin{aligned}
& \left(1+r^{2}\right)^{2-\frac{\delta}{2}}\left|\left(\Delta u_{1}\right)^{2}+\Delta\left(\left|\nabla u_{1}\right|^{2}\right)+2 \nabla u_{1} \cdot \nabla\left(\Delta u_{1}\right)\right| \leq c\left(1+r^{2}\right)^{-\frac{\delta}{2}}, \\
& \left.\left(1+r^{2}\right)^{2-\frac{\delta}{2}}\left|\Delta u_{1}\right| \nabla u_{1}\right|^{2}+\nabla u_{1} \cdot \nabla\left(\left|\nabla u_{1}\right|^{2}\right) \mid \leq c\left(1+r^{2}\right)^{-1-\frac{\delta}{2}}
\end{aligned}
$$

and

$$
\left(1+r^{2}\right)^{2-\frac{\delta}{2}}\left|\nabla u_{1}\right|^{4} \leq c\left(1+r^{2}\right)^{-\frac{\delta}{2}}
$$

This implies that given $\kappa>0$, there exists $c_{\kappa}>0$ (which can depend only on $\kappa$ ) such that, for $\delta \in(0,1)$ and $|x|=r$, we have

$$
\begin{aligned}
& \sup _{r \leq R_{\varepsilon, \lambda}}\left(1+r^{2}\right)^{2-\frac{\delta}{2}}|\mathscr{R}(0)| \\
& \quad \leq c_{\kappa} \sup _{r \leq R_{\varepsilon, \lambda}}\left(1+r^{2}\right)^{2-\frac{\delta}{2}}\left(\lambda+\frac{384 \varepsilon^{8(1-\gamma)}}{\left(\left(1+\varepsilon^{2}\right) \tau\right)^{4(1-\gamma)}\left(1+r^{2}\right)^{4 \gamma}}\right)
\end{aligned}
$$




$$
\begin{aligned}
& \leq c_{\kappa}\left(\lambda+\max \left\{\varepsilon^{8(1-\gamma)}, \varepsilon^{4+\delta} r_{\varepsilon, \lambda}^{-4-\delta}\right\}\right) \\
& \leq 2 c_{\kappa} r_{\varepsilon, \lambda}^{2} .
\end{aligned}
$$

So

$$
\|\mathscr{N}(0)\|_{\mathcal{C}_{\mathrm{rad}, \delta}^{4, \alpha}\left(\mathbb{R}^{4}\right)} \leq c_{\kappa} r_{\varepsilon, \lambda}^{2}
$$

Making use of Proposition 1 together with (19), we deduce that

$$
\|h\|_{\mathcal{C}_{\text {rad, } \delta}^{4, \alpha}\left(\mathbb{R}^{4}\right)} \leq 2 c_{\kappa} r_{\varepsilon, \lambda}^{2}
$$

Now let $h_{1}, h_{2}$ in $B\left(0,2 c_{\kappa} r_{\varepsilon, \lambda}^{2}\right)$ of $\mathcal{C}_{\mathrm{rad}, \delta}^{4, \alpha}\left(\mathbb{R}^{4}\right)$ and for $\delta \in(0,1)$, then

$$
\begin{aligned}
\sup _{r \leq R_{\varepsilon, \lambda}} & r^{4-\delta}\left|\mathscr{R}\left(h_{2}\right)-\mathscr{R}\left(h_{1}\right)\right| \\
\leq & \sup _{r \leq R_{\varepsilon, \lambda}} r^{4-\delta}\left(1+|x|^{2}\right)^{-4}\left|e^{h_{2}}-e^{h_{1}}+h_{1}-h_{2}\right| \\
& +\sup _{r \leq R_{\varepsilon, \lambda}} r^{4-\delta}\left|\mathscr{Q}_{\lambda}\left(u_{1}+h_{2}\right)-\mathscr{Q}_{\lambda}\left(u_{1}+h_{1}\right)\right| \\
& +\sup _{r \leq R_{\varepsilon, \lambda}} r^{4-\delta} \varepsilon^{8(1-\gamma)}\left(1+|x|^{2}\right)^{-4 \gamma}\left|e^{\gamma h_{2}}-e^{\gamma h_{1}}\right| .
\end{aligned}
$$

Furthermore,

$$
\begin{aligned}
& \sup _{r \leq R_{\varepsilon, \lambda}} r^{4-\delta}\left(1+|x|^{2}\right)^{-4}\left|e^{h_{2}}-e^{h_{1}}+h_{1}-h_{2}\right| \\
& \leq c \sup _{r \leq R_{\varepsilon, \lambda}} r^{4-\delta}\left(1+|x|^{2}\right)^{-4}\left|h_{2}-h_{1}\right|\left|h_{2}+h_{1}\right| \\
& \quad \leq c_{\kappa} r^{\delta} r_{\varepsilon, \lambda}^{2}\left\|h_{2}-h_{1}\right\|_{\mathcal{C}_{\text {rad }, \delta}^{4, \alpha}\left(\mathbb{R}^{4}\right)^{.}}
\end{aligned}
$$

$$
\begin{aligned}
& \sup _{r \leq R_{\varepsilon, \lambda}} r^{4-\delta} \varepsilon^{8(1-\gamma)}\left(1+|x|^{2}\right)^{-4}\left|e^{\gamma h_{2}}-e^{\gamma h_{1}}\right| \\
& \quad \leq c \sup _{r \leq R_{\varepsilon, \lambda}} r^{4-\delta} \varepsilon^{8(1-\gamma)}\left(1+|x|^{2}\right)^{-4}\left|h_{2}-h_{1}\right| \\
& \quad \leq c_{\kappa} \max \left\{\varepsilon^{8(1-\gamma)}, \varepsilon^{4} r_{\varepsilon, \lambda}^{-4}\right\}\left\|h_{2}-h_{1}\right\|_{\mathcal{C}_{\text {rad }, \delta}^{4, \alpha}\left(\mathbb{R}^{4}\right)^{.}}
\end{aligned}
$$

$$
\begin{aligned}
& r^{4-\delta}\left|\left(\Delta\left(u_{1}+h_{1}\right)\right)^{2}-\left(\Delta\left(u_{1}+h_{2}\right)\right)^{2}\right| \\
& \quad=r^{4-\delta}\left|\left(\Delta\left(h_{1}-h_{2}\right)\right)\left(\Delta\left(2 u_{1}+h_{1}+h_{2}\right)\right)\right| \\
& \quad \leq c_{\kappa}\left(1+r^{\delta} r_{\varepsilon, \lambda}^{2}\right)\left\|h_{2}-h_{1}\right\|_{\mathcal{C}_{\mathrm{rad}, \delta}^{4, \alpha}\left(\mathbb{R}^{4}\right)} .
\end{aligned}
$$




$$
\begin{aligned}
& \left.r^{4-\delta}|\Delta| \nabla\left(u_{1}+h_{2}\right)\right|^{2}-\Delta\left|\nabla\left(u_{1}+h_{1}\right)\right|^{2} \mid \\
& \quad=r^{4-\delta}\left|\Delta\left(\nabla\left(h_{1}-h_{2}\right) \cdot \nabla\left(2 u_{1}+h_{1}+h_{2}\right)\right)\right| \\
& \quad \leq c_{\kappa}\left(1+r^{\delta} r_{\varepsilon, \lambda}^{2}\right)\left\|h_{2}-h_{1}\right\|_{\mathcal{C}_{\mathrm{rad}, \delta}^{4, \alpha}\left(\mathbb{R}^{4}\right)^{4}}
\end{aligned}
$$

- $r^{4-\delta}\left|\nabla\left(\Delta\left(u_{1}+h_{2}\right)\right) \cdot \nabla\left(u_{1}+h_{2}\right)-\nabla\left(\Delta\left(u_{1}+h_{1}\right)\right) \cdot \nabla\left(u_{1}+h_{1}\right)\right|=$

$r^{4-\delta}\left|\nabla\left(\Delta\left(h_{1}-h_{2}\right)\right) \cdot \nabla\left(2 u_{1}+h_{1}+h_{2}\right)+\nabla\left(h_{2}-h_{1}\right) \cdot \nabla\left(\Delta\left(2 u_{1}+h_{1}+h_{2}\right)\right)\right| r^{4-\delta} \mid \nabla\left(\Delta\left(u_{1}+\right.\right.$

$\left.\left.h_{2}\right)\right) \cdot \nabla\left(u_{1}+h_{2}\right)-\nabla\left(\Delta\left(u_{1}+h_{1}\right)\right) \cdot \nabla\left(u_{1}+h_{1}\right) \mid \leq c_{\kappa}\left(1+r^{\delta} r_{\varepsilon, \lambda}^{2}\right)\left\|h_{2}-h_{1}\right\|_{\mathcal{C}_{\text {rad }, \delta}^{4, \alpha}\left(\mathbb{R}^{4}\right)}$.

- Since

$$
\begin{aligned}
& \left|\nabla\left(u_{1}+h_{1}\right)\right|^{2} \Delta\left(u_{1}+h_{1}\right)-\left|\nabla\left(u_{1}+h_{2}\right)\right|^{2} \Delta\left(u_{1}+h_{2}\right) \\
& =\Delta\left(h_{1}-h_{2}\right)\left[\left|\nabla\left(u_{1}+h_{1}\right)\right|^{2}+\left|\nabla\left(u_{1}+h_{2}\right)\right|^{2}\right] \\
& \quad+\Delta\left(2 u_{1}+h_{1}+h_{2}\right)\left[\left|\nabla\left(u_{1}+h_{1}\right)\right|^{2}-\left|\nabla\left(u_{1}+h_{2}\right)\right|^{2}\right],
\end{aligned}
$$

then

$$
\begin{aligned}
& \left.r^{4-\delta}|| \nabla\left(u_{1}+h_{1}\right)\right|^{2} \Delta\left(u_{1}+h_{1}\right)-\left|\nabla\left(u_{1}+h_{2}\right)\right|^{2} \Delta\left(u_{1}+h_{2}\right) \mid \\
& \quad \leq c_{\kappa}\left(1+r^{\delta} r_{\varepsilon, \lambda}^{2}+r^{2 \delta} r_{\varepsilon, \lambda}^{4}\right)\left\|h_{2}-h_{1}\right\|_{\mathcal{C}_{\mathrm{rad}, \delta}^{4, \alpha}\left(\mathbb{R}^{4}\right)} .
\end{aligned}
$$

- It is easy to see that

$$
\begin{aligned}
& \nabla\left(\left|\nabla\left(u_{1}+h_{2}\right)\right|^{2}\right) \nabla\left(u_{1}+h_{2}\right)-\nabla\left(\left|\nabla\left(u_{1}+h_{1}\right)\right|^{2}\right) \nabla\left(u_{1}+h_{1}\right) \\
& =\nabla\left(h_{2}-h_{1}\right) \nabla\left(\left|\nabla\left(u_{1}+h_{2}\right)\right|^{2}+\left|\nabla\left(u_{1}+h_{1}\right)\right|^{2}\right) \\
& \quad+\nabla\left(2 u_{1}+h_{1}+h_{2}\right) \nabla\left(\left|\nabla\left(u_{1}+h_{2}\right)\right|^{2}-\left|\nabla\left(u_{1}+h_{1}\right)\right|^{2}\right) .
\end{aligned}
$$

Hence,

$$
\begin{aligned}
& r^{4-\delta}\left|\nabla\left(\left|\nabla\left(u_{1}+h_{2}\right)\right|^{2}\right) \nabla\left(u_{1}+h_{2}\right)-\nabla\left(\left|\nabla\left(u_{1}+h_{1}\right)\right|^{2}\right) \nabla\left(u_{1}+h_{1}\right)\right| \\
& \quad \leq c_{\kappa}\left(1+r^{\delta} r_{\varepsilon, \lambda}^{2}+r^{2 \delta} r_{\varepsilon, \lambda}^{4}\right)\left\|h_{2}-h_{1}\right\|_{\mathcal{C}_{\text {rad }, \delta}^{4, \alpha}\left(\mathbb{R}^{4}\right)^{.}}
\end{aligned}
$$

- Finally, since

$$
\begin{aligned}
& \left|\nabla\left(u_{1}+h_{2}\right)\right|^{4}-\left|\nabla\left(u_{1}+h_{1}\right)\right|^{4} \\
& \quad=\nabla\left(h_{2}-h_{1}\right) \nabla\left(2 u_{1}+h_{2}+h_{1}\right)\left(\left|\nabla\left(u_{1}+h_{2}\right)\right|^{2}+\left|\nabla\left(u_{1}+h_{1}\right)\right|^{2}\right),
\end{aligned}
$$

then

$$
\begin{aligned}
& \left.r^{4-\delta}|| \nabla\left(u_{1}+h_{2}\right)\right|^{4}-\left|\nabla\left(u_{1}+h_{1}\right)\right|^{4} \mid \\
& \quad \leq c_{\kappa}\left(1+r^{\delta} r_{\varepsilon, \lambda}^{2}+r^{2 \delta} r_{\varepsilon, \lambda}^{4}+r^{3 \delta} r_{\varepsilon, \lambda}^{6}\right)\left\|h_{2}-h_{1}\right\|_{\mathcal{C}_{\mathrm{rad}, \delta}^{4, \alpha}\left(\mathbb{R}^{4}\right)} .
\end{aligned}
$$


Besides, thanks to condition $\left(A_{\lambda}\right)$ we deduce that

$$
\sup _{r \leq R_{\varepsilon, \lambda}} r^{4-\delta}\left|\mathscr{R}\left(h_{2}\right)-\mathscr{R}\left(h_{1}\right)\right| \leq c_{\kappa} r_{\varepsilon, \lambda}^{2}\left\|h_{2}-h_{1}\right\|_{\mathcal{C}_{\text {rad }, \delta}^{4, \alpha}\left(\mathbb{R}^{4}\right)} .
$$

Similarly, making use of Proposition 1 together with (19), we conclude that, given $\kappa>0$, there exist $\bar{c}_{\kappa}>0$ (independent of $\varepsilon$ and $\lambda$ ), $\lambda_{\kappa}$, and $\varepsilon_{\kappa}$ such that

$$
\left\|\mathscr{N}\left(h_{2}\right)-\mathscr{N}\left(h_{1}\right)\right\|_{\mathcal{C}_{\mathrm{rad}, \delta}^{4, \alpha}\left(\mathbb{R}^{4}\right)} \leq \bar{c}_{\kappa} r_{\varepsilon, \lambda}^{2}\left\|h_{2}-h_{1}\right\|_{\mathcal{C}_{\mathrm{rad}, \delta}^{4, \alpha}\left(\mathbb{R}^{4}\right)} .
$$

Reducing $\lambda_{\kappa}>0$ and $\varepsilon_{\kappa}>0$ if necessary, we can assume that $\bar{c}_{\kappa} r_{\varepsilon, \lambda}^{2} \leq \frac{1}{2}$ for all $\lambda \in\left(0, \lambda_{\kappa}\right)$ and $\varepsilon \in\left(0, \varepsilon_{\kappa}\right)$. Then (24) and (23) are enough to show that $h \longmapsto \mathscr{N}(h)$ is a contraction from $\left\{h \in \mathcal{C}_{\text {rad }, \delta}^{4, \alpha}\left(\mathbb{R}^{4}\right):\|h\|_{\mathcal{C}_{\text {rad }, \delta}^{4, \alpha}\left(\mathbb{R}^{4}\right)} \leq 2 c_{\kappa} r_{\varepsilon, \lambda}^{2}\right\}$ into itself and hence has a unique fixed point $h$ in this set. This fixed point is a solution of (20) in $\bar{B}_{R_{\varepsilon, \lambda}}$. We summarize this in the following proposition.

Proposition 2 Given $\delta \in(0,1)$ and $\kappa>0$, there exist $\varepsilon_{\kappa}>0, \lambda_{\kappa}>0$, and $\bar{c}_{\kappa}>0$ (depending on $\kappa)$ such that, for all $\lambda \in\left(0, \lambda_{\kappa}\right)$ and for $\varepsilon \in\left(0, \varepsilon_{\kappa}\right)$, there exists a unique solution $h \in$ $\mathcal{C}_{\text {rad, }, \delta}^{4, \alpha}\left(\mathbb{R}^{4}\right)$ of $(20)$ such that

$$
v(x)=u_{1}(x)+h(x)
$$

solves (17) in $\bar{B}_{R_{\varepsilon, \lambda}}$. In addition,

$$
\|h\|_{\mathcal{C}_{\text {rad }, \delta}^{4, \alpha}\left(\mathbb{R}^{4}\right)} \leq 2 c_{\kappa} r_{\varepsilon, \lambda}^{2} .
$$

\subsection{Analysis of the bi-Laplace operator in weighted spaces}

In this section, we prove a surjectivity result of the bi-Laplace operator in some weighted spaces and recall some estimations concerning the bi-harmonic extensions.

First, given $x^{1}, \ldots, x^{m} \in \Omega$, we define

$$
\bar{\Omega}^{*}:=\bar{\Omega}-\left\{x^{1}, \ldots, x^{m}\right\},
$$

and we choose $r_{0}>0$ so that the balls $B_{r_{0}}\left(x^{i}\right)$ of center $x^{i}$ and radius $r_{0}$ are mutually disjoint and included in $\Omega$. Given $k \in \mathbb{N}, \alpha \in(0,1)$, and $v \in \mathbb{R}$, we introduce the Hölder weighted space $\mathcal{C}_{v}^{k, \alpha}\left(\bar{\Omega}^{*}\right)$ as the space of functions $w \in \mathcal{C}_{\text {loc }}^{k, \alpha}\left(\bar{\Omega}^{*}\right)$ which is endowed with the norm

$$
\|w\|_{\mathcal{C}_{v}^{k, \alpha}\left(\bar{\Omega}^{*}\right)}:=\|w\|_{\mathcal{C}^{k, \alpha}\left(\bar{\Omega}-\bigcup_{j=1}^{m} B_{r_{0} / 2}\left(x^{j}\right)\right)}+\sum_{j=1}^{m} \sup _{0<r \leq r_{0} / 2} r^{-v}\left\|w\left(x^{j}+r \cdot\right)\right\|_{\mathcal{C}^{k, \alpha}\left(B_{2}-B_{1}\right)} .
$$

When $k \geq 2$, we let $\left[\mathcal{C}_{v}^{k, \alpha}\left(\bar{\Omega}^{*}\right)\right]_{0}$ be the subspace of functions $w \in \mathcal{C}_{v}^{k, \alpha}\left(\bar{\Omega}^{*}\right)$ satisfying $w=$ $\Delta w=0$.

In the rest of the paper, we need the following mapping properties of $\Delta^{2}$.

Proposition 3 ([4]) Assume that $v<0$ and $v \notin \mathbb{Z}$, then

$$
\Delta^{2}:\left[\mathcal{C}_{v}^{4, \alpha}\left(\bar{\Omega}^{*}\right)\right]_{0} \rightarrow \mathcal{C}_{v-4}^{0, \alpha}\left(\bar{\Omega}^{*}\right)
$$

is surjective. 
Remark 1 ([4]) It will be interesting to observe that when $v<0, v \notin \mathbb{Z}$, the right inverse, even though it is not unique, can be chosen to depend smoothly on the points $x^{1}, \ldots, x^{m}$, at least locally. Once a right inverse is fixed for some choice of the points $x^{1}, \ldots, x^{m}$, a right inverse which depends smoothly on the points $\tilde{x}^{1}, \ldots, \tilde{x}^{m}$ close to $x^{1}, \ldots, x^{m}$ can be obtained using a simple perturbation argument.

Proof Indeed, given $\left(\tilde{x}^{i}\right)$ close enough to $\left(x^{i}\right)$, we can define a family of diffeomorphisms $D: \Omega \rightarrow \Omega$ depending smoothly on $\left(\tilde{x}^{i}\right)$ by

$$
D(x)=x+\sum_{j=1}^{m} \chi_{r_{0}}\left(x-x^{j}\right)\left(x^{j}-\tilde{x}^{j}\right)
$$

where $\chi_{r_{0}}$ is a cut-off function identically equal to $1 \mathrm{in} B_{r_{0} / 2}$ and identically equal to 0 outside $B_{r_{0}}$. Hence $D\left(\tilde{x}^{j}\right)=x^{j}$ for each $j$. Then the equation $\Delta^{2} \tilde{w}=\tilde{f}$ where $\tilde{f} \in \mathcal{C}_{\nu-4}^{0, \alpha}(\bar{\Omega}-$ $\left.\left\{\tilde{x}^{i}, 1 \leq i \leq m\right\}\right)$ can be solved by considering $\tilde{w}=w \circ D$, where $w$ is a solution of the problem

$$
\Delta^{2} w+\left[\Delta^{2}(w \circ D)-\Delta^{2} w \circ D\right] \circ D^{-1}=\tilde{f} \circ D^{-1}
$$

and this time $\tilde{f} \circ D^{-1} \in \mathcal{C}_{v}^{0, \alpha}\left(\bar{\Omega}-\left\{x^{1}, \ldots, x^{m}\right\}\right)$. It should be clear that

$$
\left\|\left[\Delta^{2}(w \circ D)-\Delta^{2} w \circ D\right] \circ D^{-1}\right\|_{\mathcal{C}_{v-4}^{0, \alpha}\left(\bar{\Omega}^{*}\right)} \leq C\|w\|_{\mathcal{C}_{v}^{4, \alpha}\left(\bar{\Omega}^{*}\right)} \times \sup _{j=1, \ldots, m}\left|\tilde{x}^{j}-x^{j}\right| .
$$

Since we have a fixed right inverse for $\Delta^{2}: \mathcal{C}_{v}^{4, \alpha}\left(\bar{\Omega}^{*}\right) \rightarrow \mathcal{C}_{\nu-4}^{0, \alpha}\left(\bar{\Omega}^{*}\right)$, a perturbation argument shows that (25) is solvable provided the $\tilde{x}^{j}$ are close enough to the $x^{j}$. This provides a right inverse which depends smoothly on the choice of the points $\tilde{x}^{i}$.

\subsection{Bi-harmonic extensions}

Now, we give some estimates about the bi-harmonic extensions. More precisely, given $\varphi \in \mathcal{C}^{4, \alpha}\left(S^{3}\right)$ and $\psi \in \mathcal{C}^{2, \alpha}\left(S^{3}\right)$, we define $H^{i}\left(=H_{\varphi, \psi}^{i}\right)$ to be the solution of

$$
\begin{cases}\Delta^{2} H^{i}=0 & \text { in } B_{1}, \\ H^{i}=\varphi & \text { on } \partial B_{1}, \\ \Delta H^{i}=\psi & \text { on } \partial B_{1},\end{cases}
$$

where, as already mentioned, $B_{1}$ denotes the unit ball in $\mathbb{R}^{4}$. Given $k \in \mathbb{N}, \alpha \in(0,1)$, and $v \in$ $\mathbb{R}$, we introduce the Hölder weighted spaces $\mathcal{C}_{v}^{k, \alpha}\left(\bar{B}_{1}^{*}\right)$ as the space of function in $\mathcal{C}_{\text {loc }}^{k, \alpha}\left(\bar{B}_{1}^{*}\right)$ for which the following norm

$$
\|u\|_{\mathcal{C}_{v}^{k, \alpha}\left(\bar{B}_{1}^{*}\right)}=\sup _{r \leq 1 / 2} r^{-v}\|u(r \cdot)\|_{\mathcal{C}^{k, \alpha}\left(\bar{B}_{2}-B_{1}\right)}
$$

is finite. Here $\bar{B}_{1}^{*}=\bar{B}_{1}-\{0\}$, therefore this norm corresponds to the norm already defined in the previous section when $\Omega=B_{1}, m=1$, and $x^{1}=0$. We denote by $e_{1}, \ldots, e_{4}$ the coordinate functions on $S^{3}$. 
Lemma 2 ([4]) Assume that

$$
\int_{S^{3}}(8 \varphi-\psi) d \sigma=0 \text { and also that } \int_{S^{3}}(12 \varphi-\psi) e_{\ell} d \sigma=0
$$

for $\ell=1, \ldots, 4$. Then there exists $c>0$ such that

$$
\left\|H_{\varphi, \psi}^{i}\right\|_{\mathcal{C}_{2}^{4, \alpha}\left(\bar{B}_{1}^{*}\right)} \leq c\left(\|\varphi\|_{\mathcal{C}^{4, \alpha}\left(S^{3}\right)}+\|\psi\|_{\mathcal{C}^{2, \alpha}\left(S^{3}\right)}\right) .
$$

Also, given $\varphi \in \mathcal{C}^{4, \alpha}\left(S^{3}\right)$ and $\psi \in \mathcal{C}^{2, \alpha}\left(S^{3}\right)$, we define (when it exists !) $H^{e}\left(=H_{\varphi, \psi}^{e}\right)$ to be a solution of

$$
\begin{cases}\Delta^{2} H^{e}=0 & \text { in } \mathbb{R}^{4}-B_{1}, \\ H^{e}=\varphi & \text { on } \partial B_{1}, \\ \Delta H^{e}=\psi & \text { on } \partial B_{1},\end{cases}
$$

which decays at infinity. Given $k \in \mathbb{N}, \alpha \in(0,1)$, and $\mu \in \mathbb{R}$, we introduce the Hölder weighted spaces $\mathcal{C}_{\mu}^{k, \alpha}\left(\mathbb{R}^{4}-B_{1}\right)$ as the space of function $w \in \mathcal{C}_{\text {loc }}^{k, \alpha}\left(\mathbb{R}^{4}-B_{1}\right)$ for which the following norm

$$
\|w\|_{\mathcal{C}_{\mu}^{k, \alpha}\left(\mathbb{R}^{4}-B_{1}\right)}=\sup _{r \geq 1} r^{-\mu}\|w(r \cdot)\|_{\mathcal{C}_{\mu}^{k, \alpha}\left(\bar{B}_{2}-B_{1}\right)}
$$

is finite.

Lemma 3 ([4]) Assume that

$$
\int_{S^{3}} \psi d \sigma=0
$$

Then there exists $c>0$ such that

$$
\left\|H_{\varphi, \psi}^{e}\right\|_{\mathcal{C}_{-1}^{4, \alpha}\left(\mathbb{R}^{4}-B_{1}\right)} \leq c\left(\|\varphi\|_{\mathcal{C}^{4, \alpha}\left(S^{3}\right)}+\|\psi\|_{\mathcal{C}^{2, \alpha}\left(S^{3}\right)}\right) .
$$

Observe that, under the hypothesis of the lemma, there is uniqueness of the bi-harmonic extension of the boundary data which decays at infinity.

If $E \subset L^{2}\left(S^{3}\right)$ is a space of functions defined on $S^{3}$, we define the space $E^{\perp}$ to be the subspace of functions which are $L^{2}$-orthogonal to the functions $1, e_{1}, \ldots, e_{4}$.

Lemma 4 ([4]) The mapping

$$
\begin{aligned}
& \mathcal{P}: \mathcal{C}^{4, \alpha}\left(S^{3}\right)^{\perp} \times \mathcal{C}^{2, \alpha}\left(S^{3}\right)^{\perp} \longrightarrow \mathcal{C}^{3, \alpha}\left(S^{3}\right)^{\perp} \times \mathcal{C}^{1, \alpha}\left(S^{3}\right)^{\perp}, \\
&(\varphi, \psi) \longmapsto\left(\partial_{r}\left(H_{\varphi, \psi}^{i}-H_{\varphi, \psi}^{e}\right), \partial_{r}\left(\Delta H_{\varphi, \psi}^{i}-\Delta H_{\varphi, \psi}^{e}\right)\right)
\end{aligned}
$$




\section{The nonlinear interior problem}

We are interested in studying equations of type

$$
\Delta^{2} w+\mathscr{Q}_{\lambda}(w)-24\left(e^{w}+\frac{2^{4(1-\gamma)} \varepsilon^{8(1-\gamma)}}{\left(\left(1+\varepsilon^{2}\right) \tau\right)^{4(1-\gamma)}} e^{\gamma w}\right)=0
$$

in $\bar{B}_{R_{\varepsilon, \lambda}}$.

Given $\varphi \in \mathcal{C}^{4, \alpha}\left(S^{3}\right)$ and $\psi \in \mathcal{C}^{2, \alpha}\left(S^{3}\right)$. Let $\kappa>0$ (whose value will be fixed later on), we further assume that the functions $\varphi, \psi$ satisfy

$$
\|\varphi\|_{\mathcal{C}^{4, \alpha}} \leq \kappa r_{\varepsilon, \lambda}^{2} \quad \text { and } \quad\|\psi\|_{\mathcal{C}^{2, \alpha}} \leq \kappa r_{\varepsilon, \lambda}^{2} .
$$

Define

$$
\mathbf{v}:=u_{1}+H^{i}\left(\varphi, \psi, \cdot / R_{\varepsilon, \lambda}\right)+h,
$$

then we look for a solution of (28) of the form $w=\mathbf{v}+v$, and using the fact that $H^{i}$ is biharmonic, this amounts to solving

$$
\begin{aligned}
\mathscr{L} v= & \frac{384}{\left(1+|x|^{2}\right)^{4}} e^{h}\left(e^{H^{i}\left(\varphi, \psi, \cdot / R_{\varepsilon, \lambda}\right)+v}-v-1\right)+\frac{384}{\left(1+|x|^{2}\right)^{4}}\left(e^{h}-1\right) v \\
& +\frac{384 \varepsilon^{8(1-\gamma)}}{\left(\left(1+\varepsilon^{2}\right) \tau\right)^{4(1-\gamma)}\left(1+|x|^{2}\right)^{4 \gamma}} e^{\gamma h}\left(e^{\gamma\left(H^{i}\left(\varphi, \psi, \cdot / R_{\varepsilon, \lambda}\right)+v\right)}-1\right) \\
& +\mathscr{Q}_{\lambda}\left(u_{1}+h\right)-\mathscr{Q}_{\lambda}\left(u_{1}+H^{i}\left(\varphi, \psi, \cdot / R_{\varepsilon, \lambda}\right)+h+v\right) .
\end{aligned}
$$

We fix

$$
\mu \in(1,2)
$$

and denote by $\mathscr{G}_{\mu}$ a right inverse of $\mathscr{L}_{\mu}$ provided by Proposition 1 . To obtain a solution of (30), it is sufficient to find $v \in \mathcal{C}_{\mu}^{4, \alpha}\left(\mathbb{R}^{4}\right)$ solution of

$$
v=\mathscr{N}(v):=\mathscr{G}_{\mu} \circ \mathcal{E}_{\mu} \circ \mathscr{S}(v),
$$

where

$$
\begin{aligned}
\mathscr{S}(v):= & \frac{384}{\left(1+|x|^{2}\right)^{4}} e^{h}\left(e^{H^{i}\left(\varphi, \psi, / / R_{\varepsilon, \lambda}\right)+v}-v-1\right)+\frac{384}{\left(1+|x|^{2}\right)^{4}}\left(e^{h}-1\right) v \\
& +\frac{384 \varepsilon^{8(1-\gamma)}}{\left(\left(1+\varepsilon^{2}\right) \tau\right)^{4(1-\gamma)}\left(1+|x|^{2}\right)^{4 \gamma}} e^{\gamma h}\left(e^{\gamma\left(H^{i}\left(\varphi, \psi, / R_{\varepsilon, \lambda}\right)+v\right)}-1\right) \\
& +\mathscr{Q}_{\lambda}\left(u_{1}+h\right)-\mathscr{Q}_{\lambda}\left(u_{1}+H^{i}\left(\varphi, \psi, \cdot / R_{\varepsilon, \lambda}\right)+h+v\right) .
\end{aligned}
$$

We denote by $\mathscr{N}\left(=\mathscr{N}_{\varepsilon, \lambda, \varphi, \psi}\right)$ the nonlinear operator appearing on the right-hand side of Eq. (31), then we have the following result. 
Lemma 5 Given $\mu \in(1,2)$ and $\kappa>0$, there exist $\lambda_{\kappa}>0, \varepsilon_{\kappa}>0, c_{\kappa}>0$, and $\bar{c}_{\kappa}>0$ (depending on $\kappa)$ such that, for all $\lambda \in\left(0, \lambda_{\kappa}\right)$ and $\varepsilon \in\left(0, \varepsilon_{\kappa}\right)$,

$$
\|\mathscr{N}(0)\|_{\mathcal{C}_{\mu}^{4, \alpha}\left(\mathbb{R}^{4}\right)} \leq c_{\kappa} r_{\varepsilon, \lambda}^{2}
$$

Moreover,

$$
\left\|\mathscr{N}\left(v_{2}\right)-\mathscr{N}\left(v_{1}\right)\right\|_{\mathcal{C}_{\mu}^{4, \alpha}\left(\mathbb{R}^{4}\right)} \leq \bar{c}_{\kappa} r_{\varepsilon, \lambda}^{2}\left\|v_{2}-v_{1}\right\|_{\mathcal{C}_{\mu}^{4, \alpha}\left(\mathbb{R}^{4}\right)},
$$

provided that $v_{1}, v_{2} \in \mathcal{C}_{\mu}^{4, \alpha}\left(\mathbb{R}^{4}\right)$ satisfy $\left\|v_{i}\right\|_{\mathcal{C}_{\mu}^{4, \alpha}\left(\mathbb{R}^{4}\right)} \leq 2 c_{\kappa} r_{\varepsilon, \lambda}^{2}$.

Proof The proof of the first estimate follows from the asymptotic behavior of $H^{i}$ together with the assumption on the norm of boundary data $\varphi$ and $\psi$ given by (29). Indeed, let $c_{\kappa}$ be a constant depending only on $\kappa$ (provided $\varepsilon$ and $\lambda$ are chosen small enough), it follows from the estimate of $H^{i}$, given by Lemma 2 , that

$$
\left\|H^{i}\left(\varphi, \psi, \cdot / R_{\varepsilon, \lambda}\right)\right\|_{\mathcal{C}_{2}^{4, \alpha}\left(\bar{B}_{R_{\varepsilon, \lambda}}\right)} \leq c R_{\varepsilon, \lambda}^{-2}\left(\|\varphi\|_{\mathcal{C}^{4, \alpha}\left(S^{3}\right)}+\|\psi\|_{\mathcal{C}^{2, \alpha}\left(S^{3}\right)}\right) \leq c_{\kappa} \varepsilon^{2}
$$

Since, for each $x \in \bar{B}_{R_{\varepsilon, \lambda}}$, we have

$$
|h(x)| \leq c_{\kappa} r_{\varepsilon, \lambda}^{2+\delta} \varepsilon^{-\delta} \leq \begin{cases}\varepsilon^{1-\delta / 2} & \text { for } \varepsilon \geq \lambda \\ \lambda^{1+\delta / 2} \varepsilon^{-\delta} & \text { for } \lambda>\varepsilon\end{cases}
$$

Then, using condition $\left(A_{\lambda}\right)$, we prove that $|h(x)| \rightarrow 0$ as $\varepsilon$ and $\lambda$ tend to 0 . Given $\kappa>0$, there exists $c_{\kappa}>0$ such that

$$
\left\|\left(1+|\cdot|^{2}\right)^{-2} e^{h}\left(e^{H^{i}\left(\varphi, \psi, / R_{\varepsilon, \lambda}\right)}-1\right)\right\|_{\mathcal{C}_{\mu-2}^{0, \alpha}\left(\bar{B}_{R_{\varepsilon, \lambda}}\right)} \leq c_{\kappa} \varepsilon^{2} .
$$

On the other hand, using condition $\left(A_{\lambda}\right)$, we also get

$$
\sup _{r \leq R_{\varepsilon, \lambda}}\left(1+r^{2}\right)^{2-\frac{\mu}{2}}\left|\mathscr{Q}_{\lambda}\left(u_{1}+h\right)-\mathscr{Q}_{\lambda}\left(u_{1}+H^{i}\left(\varphi, \psi, \cdot / R_{\varepsilon, \lambda}\right)+h\right)\right| \leq c_{\kappa} r_{\varepsilon, \lambda}^{2}
$$

and

$$
\begin{aligned}
& \left\|\frac{384 \varepsilon^{8(1-\gamma)}}{\left(\left(1+\varepsilon^{2}\right) \tau\right)^{4(1-\gamma)}\left(1+|\cdot|^{2}\right)^{4 \gamma}} e^{\gamma h}\left(e^{\gamma\left(H^{i}\left(\varphi, \psi, \cdot / R_{\varepsilon, \lambda}\right)+\nu\right)}-1\right)\right\|_{\mathcal{C}_{\mu-2}^{0, \alpha}\left(\bar{B}_{R_{\varepsilon, \lambda}}\right)} \\
& \quad \leq c_{\kappa} \varepsilon^{2} \varepsilon^{8(1-\gamma)} \\
& \quad \leq c_{\kappa} r_{\varepsilon, \lambda}^{2} .
\end{aligned}
$$

Making use of Proposition 1 together with (19), we get for $\mu \in(1,2)$

$$
\|\mathscr{N}(0)\|_{\mathcal{C}_{\mu}^{4, \alpha}\left(\mathbb{R}^{4}\right)} \leq c_{\kappa} r_{\varepsilon, \lambda}^{2}
$$


To derive the second estimate, let $v_{i} \in \mathcal{C}_{\mu}^{4, \alpha}\left(\mathbb{R}^{4}\right)$ satisfy $\left\|v_{i}\right\|_{\mathcal{C}_{\mu}^{4, \alpha}\left(\mathbb{R}^{4}\right)} \leq 2 c_{\kappa} r_{\varepsilon, \lambda}^{2}, i=1,2, \mu \in$ $(1,2)$, and condition $A_{\lambda}$. Hence there exists $c_{\kappa}>0$ such that

$$
\begin{aligned}
& \left\|\left(1+|\cdot|^{2}\right)^{-4} e^{h}\left(e^{H^{i}\left(\varphi, \psi, \cdot / R_{\varepsilon, \lambda}\right)}\left(e^{v_{2}}-e^{v_{1}}\right)-\left(v_{2}-v_{1}\right)\right)\right\|_{\mathcal{C}_{\mu-4}^{0, \alpha}\left(\bar{B}_{R_{\varepsilon, \lambda}}\right)} \leq c_{\kappa} \varepsilon^{2}\left\|v_{2}-v_{1}\right\|_{\mathcal{C}_{\mu}^{4, \alpha}\left(\mathbb{R}^{4}\right)^{\prime}}, \\
& \left\|\left(e^{h}-1\right)\left(v_{2}-v_{1}\right)\right\|_{\mathcal{C}_{\mu-4}^{0, \alpha}\left(\bar{B}_{R_{\varepsilon, \lambda}}\right)} \leq c_{\kappa} r_{\varepsilon, \lambda}^{2}\left\|v_{2}-v_{1}\right\|_{\mathcal{C}_{\mu}^{4, \alpha}\left(\mathbb{R}^{4}\right)^{4}} \\
& \left\|\mathscr{Q}_{\lambda}\left(u_{1}+H^{i}\left(\varphi, \psi, \cdot / R_{\varepsilon, \lambda}\right)+h+v_{2}\right)-\mathscr{Q}_{\lambda}\left(u_{1}+H^{i}\left(\varphi, \psi, \cdot / R_{\varepsilon, \lambda}\right)+h+v_{1}\right)\right\|_{\mathcal{C}_{\mu-4}^{0, \alpha}\left(\bar{B}_{R_{\varepsilon, \lambda}}\right)} \\
& \quad \leq c_{\kappa} r_{\varepsilon, \lambda}^{2}\left\|v_{2}-v_{1}\right\|_{\mathcal{C}_{\mu}^{4, \alpha}\left(\mathbb{R}^{4}\right)}
\end{aligned}
$$

and

$$
\begin{aligned}
& \left\|\frac{384 \varepsilon^{8(1-\gamma)}}{\left(\left(1+\varepsilon^{2}\right) \tau\right)^{4(1-\gamma)}\left(1+|\cdot|^{2}\right)^{4 \gamma}} e^{\gamma h}\left(e^{\gamma H^{i}\left(\varphi, \psi, / / R_{\varepsilon, \lambda}\right)}\left(e^{\gamma \nu_{2}}-e^{\gamma v_{1}}\right)\right)\right\|_{\mathcal{C}_{\mu-4}^{0, \alpha}\left(\bar{B}_{R_{\varepsilon, \lambda}}\right)} \\
& \quad \leq c_{\kappa} \varepsilon^{2}\left\|v_{2}-v_{1}\right\|_{\mathcal{C}_{\mu}^{4, \alpha}\left(\mathbb{R}^{4}\right)^{.}}
\end{aligned}
$$

So

$$
\sup _{r \leq R_{\varepsilon, \lambda}}\left(1+r^{2}\right)^{2-\frac{\mu}{2}}\left|\mathscr{S}\left(v_{2}\right)-\mathscr{S}\left(v_{1}\right)\right| \leq c_{\kappa} r_{\varepsilon, \lambda}^{2}\left\|v_{2}-v_{1}\right\|_{\mathcal{C}_{\mathrm{rad}, \mu}^{4, \alpha}\left(\mathbb{R}^{4}\right)^{.}}
$$

Similarly, making use of Proposition 1 together with (19), we conclude that there exists $\bar{c}_{\kappa}>0$ such that

$$
\left\|\mathscr{N}\left(v_{2}\right)-\mathcal{N}\left(v_{1}\right)\right\|_{\mathcal{C}_{\mu}^{4, \alpha}\left(\mathbb{R}^{4}\right)} \leq \bar{c}_{\kappa} r_{\varepsilon, \lambda}^{2}\left\|v_{2}-v_{1}\right\|_{\mathcal{C}_{\mu}^{4, \alpha}\left(\mathbb{R}^{4}\right)} .
$$

Reducing $\lambda_{\kappa}>0$ and $\varepsilon_{\kappa}>0$ if necessary, we can assume that

$$
\bar{c}_{\kappa} r_{\varepsilon, \lambda}^{2} \leq \frac{1}{2}
$$

for all $\lambda \in\left(0, \lambda_{\kappa}\right)$ and $\varepsilon \in\left(0, \varepsilon_{\kappa}\right)$. Then (33) and (34) in Lemma 5 are enough to show that

$$
v \longmapsto \mathscr{N}(v)
$$

is a contraction from

$$
\left\{v \in \mathcal{C}_{\mu}^{4, \alpha}\left(\mathbb{R}^{4}\right):\|v\|_{\mathcal{C}_{\mu}^{4, \alpha}\left(\mathbb{R}^{4}\right)} \leq 2 c_{\kappa} \varepsilon^{2}\right\}
$$

into itself and hence has a unique fixed point $v=v(\varepsilon, \tau, \varphi, \psi ; \cdot)$ in this set. This fixed point is a solution of (31) in $\mathbb{R}^{4}$. We summarize this in the following proposition.

Proposition 4 Given $\mu \in(1,2)$ and $\kappa>0$, there exist $\varepsilon_{\kappa}>0, \lambda_{\kappa}>0$, and $c_{\kappa}>0$ (depending on $\kappa)$ such that, for all $\varepsilon \in\left(0, \varepsilon_{\kappa}\right), \lambda \in\left(0, \lambda_{\kappa}\right)$ satisfying $\left(A_{\lambda}\right)$, for all $\tau$ in some fixed compact subset of $\left[\tau_{-}, \tau^{+}\right] \subset(0, \infty)$, and for given $\varphi$ and $\psi$ satisfying (26)-(29), then there exists a unique $v\left(:=\bar{v}_{\varepsilon, \tau, \varphi, \psi}\right)$ solution of $(31)$ such that

$$
w:=u_{1}+H^{i}\left(\varphi, \psi, \cdot / R_{\varepsilon, \lambda}\right)+h+\bar{v}_{\varepsilon, \tau, \varphi, \psi}
$$


solves (28) in $\bar{B}_{R_{\varepsilon, \lambda}}$. In addition,

$$
\|v\|_{\mathcal{C}_{\mu}^{4, \alpha}\left(\mathbb{R}^{4}\right)} \leq 2 c_{\kappa} r_{\varepsilon, \lambda}^{2}
$$

\section{The nonlinear exterior problem}

Denote $G_{\tilde{x}}=G(x, \tilde{x})$ where $G$ is the Green's function given by $(11)$ and $H(x, \tilde{x})$ is its regular part. Clearly $x \mapsto H(x, \tilde{x})$ is a smooth function. Let $\tilde{\mathbf{x}}=\left(\tilde{x}^{j}\right) \in \Omega^{m}$ close to $\mathbf{x}=\left(x^{j}\right), \tilde{\boldsymbol{\eta}}=\left(\tilde{\eta}^{j}\right) \in$ $\mathbb{R}^{m}$ close to 0 . Let $\tilde{\boldsymbol{\varphi}}=\left(\tilde{\varphi}^{j}\right) \in\left(\mathcal{C}^{4, \alpha}\left(S^{3}\right)\right)^{m}$ and $\tilde{\boldsymbol{\psi}}=\left(\tilde{\psi}^{j}\right) \in\left(\mathcal{C}^{2, \alpha}\left(S^{3}\right)\right)^{m}$ satisfy (27). We define

$$
\tilde{\mathbf{u}}=\tilde{u}_{\varepsilon, \tilde{\eta}, \tilde{\mathbf{x}}, \tilde{\varphi}, \tilde{\psi}}:=\sum_{j=1}^{m}\left(1+\tilde{\eta}^{j}\right) G_{\tilde{x}^{j}}+\sum_{j=1}^{m} \chi_{r_{0}}\left(x-\tilde{x}^{j}\right) H_{\tilde{\varphi}^{\prime}, \tilde{\psi}^{j}}^{e}\left(\frac{x-\tilde{x}^{j}}{r_{\varepsilon, \lambda}}\right)
$$

where $\chi_{r_{0}}$ is a cut-off function identically equal to 1 in $B_{r_{0} / 2}$ and identically equal to 0 outside $B_{r_{0}}$. We would like to solve the equation

$$
\Delta^{2} u+\mathscr{Q}_{\lambda}(u)-\rho^{4}\left(e^{u}+e^{\gamma u}\right)=0, \quad \text { in } \Omega-\bigcup_{1 \leq j \leq m} B_{r_{\varepsilon, \lambda}(\tilde{x})},
$$

with $u=\tilde{\mathbf{u}}+\tilde{v}$ is a perturbation of $\tilde{\mathbf{u}}$. This amounts to solving

$$
\Delta^{2} \tilde{v}=\rho^{4}\left(e^{\tilde{\mathbf{u}}} e^{\tilde{v}}-e^{\gamma \tilde{\mathbf{u}}} e^{\gamma \tilde{v}}\right)-\mathscr{Q}_{\lambda}(\tilde{\mathbf{u}}+\tilde{v})-\Delta^{2} \tilde{\mathbf{u}}=\tilde{\mathscr{S}}(\tilde{v}) .
$$

Denote $\Omega_{R, \tilde{x}}=\Omega-\bigcup_{1 \leq j \leq m} B_{R}\left(\tilde{x}^{j}\right)$ for any $R>0$. We denote by

$$
\tilde{\xi}_{R}: \mathcal{C}_{v}^{0, \alpha}\left(\bar{\Omega}_{R, \tilde{x}}\right) \longrightarrow \mathcal{C}_{v}^{0, \alpha}\left(\bar{\Omega}^{*}\right)
$$

the extension operator defined by

$$
\begin{cases}\tilde{\xi}_{R}(f) \equiv f & \text { in } \Omega_{R, \tilde{x}}, \\ \tilde{\xi}_{R}(f)\left(x_{i}+x\right)=\frac{2|x|-R}{R} f\left(x_{i}+\frac{R x}{|x|}\right) & \text { in } B_{R}\left(\tilde{x}^{j}\right) \backslash B_{R / 2}\left(\tilde{x}^{j}\right), \forall 1 \leq j \leq m, \\ \tilde{\xi}_{R}(f) \equiv 0 & \text { in } \bigcup_{j} B_{R / 2}\left(\tilde{x}^{j}\right) .\end{cases}
$$

It is easy to check that there exists a constant $c=c(v)>0$, only depending on $v$, such that

$$
\left\|\tilde{\xi_{R}}(w)\right\|_{\mathcal{C}_{v}^{0, \alpha}\left(\bar{\Omega}^{*}\right)} \leq c\|w\|_{\mathcal{C}_{v}^{0, \alpha}\left(\bar{\Omega}_{R, \tilde{x}}\right.}{ }
$$

We fix

$$
v \in(-1,0)
$$

and denote by $\tilde{\mathscr{G}}_{v}$ the right inverse provided by Proposition 3. Clearly, it is enough to find $\tilde{v} \in \mathcal{C}_{v}^{4, \alpha}\left(\Omega^{*}\right)$ solution of

$$
\tilde{v}=\tilde{\mathscr{G}}_{\nu} \circ \tilde{\xi}_{r_{\varepsilon, \lambda}} \circ \tilde{\mathscr{S}}(\tilde{v}) .
$$

We denote by $\tilde{\mathscr{N}}(\tilde{v})\left(=\tilde{\mathscr{N}}_{\varepsilon, \eta, \tilde{x}, \tilde{\varphi}, \tilde{\psi}}(\tilde{v})\right)=\tilde{\mathscr{G}}_{\nu} \circ \tilde{\xi}_{\varepsilon, \lambda} \circ \tilde{\mathscr{S}}(\tilde{v})$ the nonlinear operator on the righthand side. Even though this is not notified in the notation, $\tilde{\mathscr{G}}_{v}: \mathcal{C}_{\nu-4}^{0, \alpha}\left(\bar{\Omega}^{*}\right) \longrightarrow \mathcal{C}_{v}^{4, \alpha}\left(\bar{\Omega}^{*}\right)$ is 
the right inverse defined in Remark 1 with $\bar{\Omega}^{*}=\bar{\Omega}-\left\{\tilde{x}^{1}, \ldots, \tilde{x}^{m}\right\}$. Given $\kappa>0$ (whose value will be fixed later on), we further assume that the functions $\tilde{\varphi}^{j}$ and $\tilde{\psi}^{j}$ satisfy

$$
\left\|\tilde{\varphi}^{j}\right\|_{\mathcal{C}^{4, \alpha}} \leq \kappa r_{\varepsilon, \lambda}^{2} \quad \text { and } \quad\left\|\tilde{\psi}^{j}\right\|_{\mathcal{C}^{2, \alpha}} \leq \kappa r_{\varepsilon, \lambda}^{2}, \quad \forall j=1, \ldots, m
$$

Moreover, we assume that the parameters $\tilde{\eta}^{j}$ and the points $\tilde{x}^{j}$ are chosen to verify

$$
\left|\tilde{\eta}^{j}\right| \leq \kappa r_{\varepsilon, \lambda}^{2} \quad \text { and } \quad r_{\varepsilon, \lambda}\left|\tilde{x}^{j}-x^{j}\right| \leq \kappa r_{\varepsilon, \lambda}^{2} .
$$

Then the following result holds.

Lemma 6 Given $v \in(-1,0)$ and $\kappa>0$, there exist $\varepsilon_{\kappa}>0$ and $c_{\kappa}>0$ (depending on $\kappa$ ) such that, for all $\varepsilon \in\left(0, \varepsilon_{\kappa}\right)$ and under assumptions (40) and (41), we have

$$
\|\tilde{\mathscr{N}}(0)\|_{\mathcal{C}_{v}^{4, \alpha}\left(\bar{\Omega}^{*}\right)} \leq c_{\kappa} r_{\varepsilon, \lambda}^{2}
$$

and

$$
\left\|\tilde{\mathscr{N}}\left(\tilde{v}_{2}\right)-\tilde{\mathscr{N}}\left(\tilde{v}_{1}\right)\right\|_{\mathcal{C}_{v}^{4, \alpha}\left(\bar{\Omega}^{*}\right)} \leq \bar{c}_{\kappa} r_{\varepsilon, \lambda}^{2}\left\|\tilde{v}_{2}-\tilde{v}_{1}\right\|_{\mathcal{C}_{v}^{4, \alpha}\left(\bar{\Omega}^{*}\right)}
$$

provided that $\tilde{v}_{1}, \tilde{v}_{2} \in \mathcal{C}_{v}^{4, \alpha}\left(\bar{\Omega}^{*}\right)$ and $\left\|\tilde{v}_{i}\right\|_{\mathcal{C}_{v}^{4, \alpha}\left(\bar{\Omega}^{*}\right)} \leq 2 c_{\kappa} r_{\varepsilon, \lambda}^{2}$.

Proof The proof of the first estimate follows from the asymptotic behavior of $H^{e}$ together with the assumption on the norm of boundary data $\tilde{\varphi}_{j}$ and $\tilde{\psi}_{j}$ given by (40). Indeed, let $c_{\kappa}$ be a constant depending only on $\kappa$ (provided $\varepsilon$ and $\lambda$ are chosen small enough), it follows from the estimate of $H^{e}$, given by Lemma 3, that

$$
\left|H_{\tilde{\varphi}_{j}, \tilde{\psi}_{j}}^{e}\left(\frac{x-\tilde{x}^{j}}{r_{\varepsilon, \lambda}}\right)\right| \leq c_{\kappa} r_{\varepsilon, \lambda}^{3} r^{-1}
$$

Recall that $\tilde{\mathscr{N}}(\tilde{v})=\tilde{\mathscr{G}}_{\nu} \circ \tilde{\xi}_{r_{\varepsilon}, \lambda} \circ \tilde{\mathscr{S}}(\tilde{v})$, we will estimate $\tilde{\mathscr{N}}(0)$ in different subregions of $\bar{\Omega}^{*}$.

- In $B_{r_{0}}\left(\tilde{x}^{j}\right)$ for $1 \leq j \leq m$, we have $\chi_{r_{0}}\left(x-\tilde{x}^{j}\right)=1$ and $\Delta^{2} \tilde{\mathbf{u}}=0$, so that

$$
\begin{aligned}
& |\tilde{\mathscr{S}}(0)| \leq c \varepsilon^{4} \prod_{j=1}^{m} e^{\left(1+\tilde{n}^{j}\right) G_{\tilde{x} j}(x)+H_{\tilde{\varphi}^{j}, \tilde{\psi}^{j}}^{e}\left(\left(x-\tilde{x}^{j}\right) / r_{\varepsilon}\right)}
\end{aligned}
$$

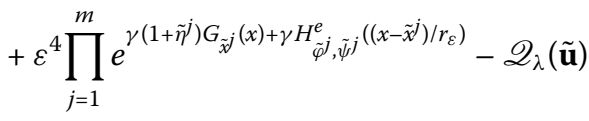

$$
\begin{aligned}
& \leq c \varepsilon^{4} \prod_{j=1}^{m}\left|x-\tilde{x}^{j}\right|^{-8\left(1+\tilde{\eta}^{j}\right)}+\varepsilon^{4} \prod_{j=1}^{m}\left|x-\tilde{x}^{j}\right|^{-8 \gamma\left(1+\tilde{\eta}^{j}\right)}+\left|\mathscr{Q}_{\lambda}(\tilde{\mathbf{u}})\right| .
\end{aligned}
$$

So, by an easy computation, for $v \in(-1,0)$ and $\tilde{\eta}^{j}$ small enough, we get

$$
\|\tilde{\mathscr{S}}(0)\|_{\mathcal{C}_{v}^{4, \alpha}\left(\bigcup_{j=1}^{m} B\left(\tilde{x}, r_{0}\right)\right)} \leq \sup _{r_{\varepsilon, \lambda} \leq r \leq r_{0} / 2} r^{4-v}|\tilde{\mathscr{S}}(0)| \leq c_{\kappa}\left(\varepsilon^{4} r_{\varepsilon, \lambda}^{-4}+\lambda\right)
$$


- In $\Omega-B_{r_{0}}\left(\tilde{x}^{j}\right)$, we have $\chi_{r_{0}}\left(x-\tilde{x}^{j}\right)=0$ and $\Delta^{2} \tilde{\mathbf{u}}=0$, then

$$
|\tilde{\mathscr{S}}(0)| \leq c\left(\varepsilon^{4} \prod_{j=1}^{m} e^{\left(1+\tilde{\eta}^{j}\right) G_{\tilde{x}}}+\varepsilon^{4} \prod_{j=1}^{m} e^{\gamma\left(1+\tilde{\eta}^{j}\right) G_{\tilde{x} j}}+\left|\mathscr{Q}_{\lambda}(\tilde{\mathbf{u}})\right|\right) .
$$

Thus

$$
\|\tilde{\mathscr{S}}(0)\|_{\mathcal{C}_{v}^{4, \alpha}\left(\Omega_{r_{0}, \tilde{x}}\right)} \leq c_{\kappa} \sup _{r \geq r_{0}} r^{4-v}|\tilde{\mathscr{S}}(0)| \leq c_{\kappa}\left(\varepsilon^{4}+\lambda\right) .
$$

- In $B_{r_{0}}\left(\tilde{x}^{j}\right)-B_{r_{0} / 2}\left(\tilde{x}^{j}\right)$, using estimate (42), we have

$$
\begin{aligned}
|\tilde{\mathscr{S}}(0)| \leq & c_{\kappa} \varepsilon^{4} \prod_{j=1}^{m}\left|x-\tilde{x}^{j}\right|^{-\left(8+\tilde{\eta}^{j}\right)}+c_{\kappa} \varepsilon^{4} \prod_{j=1}^{m}\left|x-\tilde{x}^{j}\right|^{-\gamma\left(8+\tilde{\eta}^{j}\right)}+\left|\mathscr{Q}_{\lambda}(\tilde{\mathbf{u}})\right| \\
& +c \varepsilon^{4} \sum_{j=1}^{m}\left|\left[\Delta^{2}, \chi_{r_{0}}\right]\left(x-\tilde{x}^{j}\right)\right|\left|H_{\tilde{\varphi}_{j}, \tilde{\psi}_{j}}^{\mathrm{ext}}\left(\left(x-\tilde{x}^{j}\right) / r_{\varepsilon, \lambda}\right)\right| .
\end{aligned}
$$

Here

$$
\left[\Delta^{2}, \chi_{r_{0}}\right] w=2 \Delta \chi_{r_{0}} \Delta w+w \Delta^{2} \chi_{r_{0}}+4 \nabla \chi_{r_{0}} \cdot \nabla(\Delta w)+4 \nabla w \cdot \nabla\left(\Delta \chi_{r_{0}}\right)+4 \nabla^{2} \chi_{r_{0}} \cdot \nabla^{2} w
$$

So,

$$
\|\tilde{\mathscr{S}}(0)\|_{\mathcal{C}_{v}^{4, \alpha}\left(B\left(\tilde{x}, r_{0}\right)-B\left(\tilde{x}, r_{0} / 2\right)\right)} \leq c_{\kappa} \sup _{r_{0} / 2 \leq r \leq r_{0}} r^{4-v}|\tilde{\mathscr{S}}(0)| \leq c_{\kappa}\left(r_{\varepsilon, \lambda}^{2}+\lambda\right) .
$$

Finally, making Proposition 3 with (38), we conclude that

$$
\|\tilde{\mathscr{N}}(0)\|_{\mathcal{C}_{\nu}^{4, \alpha}\left(\bar{\Omega}^{*}\right)} \leq c_{\kappa} r_{\varepsilon, \lambda}^{2}
$$

For the proof of the second estimate, let $\tilde{v}_{1}$ and $\tilde{v}_{2} \in C_{v}^{4, \alpha}\left(\bar{\Omega}^{*}\right)$ satisfying $\left\|\tilde{v}_{i}\right\|_{\mathcal{C}_{v}^{4, \alpha}} \leq c_{\kappa} r_{\varepsilon, \lambda}^{2}$, so

$$
\begin{aligned}
& \quad\left|\left(\tilde{\mathscr{S}}\left(\tilde{v}_{2}\right)-\tilde{\mathscr{S}}\left(\tilde{v}_{1}\right)\right)\right| \\
& \quad \leq c_{\kappa}\left|\rho^{4}\left(e^{\tilde{\mathbf{u}}}\left(e^{\tilde{v}_{2}}-e^{\tilde{\nu}_{1}}\right)-e^{\gamma \tilde{\mathbf{u}}}\left(e^{\gamma \tilde{v}_{2}}-e^{\gamma \tilde{v}_{1}}\right)\right)-\left(\mathscr{Q}_{\lambda}\left(\tilde{\mathbf{u}}+\tilde{v}_{2}\right)-\mathscr{Q}_{\lambda}\left(\tilde{\mathbf{u}}+\tilde{v}_{1}\right)\right)\right| .
\end{aligned}
$$

Then, for $\tilde{\eta}^{j}$ small enough and using estimate (38), there exists $\bar{c}_{\kappa}>0$ (depending on $\kappa$ ) such that

$$
\left\|\tilde{\mathscr{N}}\left(\tilde{v}_{2}\right)-\tilde{\mathscr{N}}\left(\tilde{v}_{1}\right)\right\|_{\mathcal{C}_{v}^{4, \alpha}\left(\bar{\Omega}^{*}\right)} \leq \bar{c}_{\kappa} r_{\varepsilon, \lambda}^{2}\left\|\tilde{v}_{2}-\tilde{v}_{1}\right\|_{\mathcal{C}_{v}^{4, \alpha}\left(\bar{\Omega}^{*}\right)}
$$

Then we get the second estimate.

Reducing $\lambda_{\kappa}>0$ and $\varepsilon_{\kappa}>0$ if necessary, we can assume that

$$
\bar{c}_{\kappa} r_{\varepsilon, \lambda}^{2} \leq \frac{1}{2}
$$


for all $\lambda \in\left(0, \lambda_{\kappa}\right)$ and $\varepsilon \in\left(0, \varepsilon_{\kappa}\right)$. Then (44) and (43) are enough to show that

$$
\tilde{v} \longmapsto \tilde{N}(\tilde{v})
$$

is a contraction from

$$
\left\{\tilde{v} \in \mathcal{C}_{v}^{4, \alpha}\left(\mathbb{R}^{4}\right):\|\tilde{v}\|_{\mathcal{C}_{v}^{4, \alpha}\left(\mathbb{R}^{4}\right)} \leq 2 c_{\kappa} r_{\varepsilon, \lambda}^{2}\right\}
$$

into itself and hence has a unique fixed point $\tilde{v}=\tilde{v}(\varepsilon, \tau, \varphi, \psi ; \cdot)$ in this set. This fixed point is a solution of (39) in $\mathbb{R}^{4}$. We summarize this in the following proposition.

Proposition 5 Given $v \in(-1,0)$ and $\kappa>0$, there exist $\varepsilon_{\kappa}>0, \lambda_{\kappa}>0$, and $c_{\kappa}>0$ (depending on $\kappa)$ such that, for all $\varepsilon \in\left(0, \varepsilon_{\kappa}\right)$ and $\lambda \in\left(0, \lambda_{\kappa}\right)$, for all set of parameters $\tilde{\eta}^{j}$ and points $\tilde{x}^{j}$ satisfying (41), all functions $\tilde{\varphi}^{j}, \tilde{\psi}^{j}$ satisfying (27) and (40), there exists a unique $\tilde{v}(=$ $\left.\tilde{v}_{\varepsilon, \eta, \tilde{\mathbf{x}}, \tilde{\varphi}, \tilde{\psi}}\right)$ solution of (39) such that

$$
\tilde{u}_{\varepsilon, \eta, \tilde{\mathbf{x}}, \tilde{\varphi}, \tilde{\psi}}:=\sum_{j=1}^{m}\left(1+\tilde{\eta}^{j}\right) G_{\tilde{x}^{j}}+\sum_{j=1}^{m} \chi_{r_{0}}\left(\cdot-\tilde{x}^{j}\right) H_{\tilde{\varphi}^{j}, \tilde{\psi}^{j}}^{e}\left(\frac{x-\tilde{x}^{j}}{r_{\varepsilon}}\right)+\tilde{v}_{\varepsilon, \tilde{\eta}, \tilde{x}, \tilde{\varphi}, \tilde{\psi}}
$$

solves (37) in $\bar{\Omega}^{*}$. In addition,

$$
\|\tilde{v}\|_{\mathcal{C}_{v}^{4, \alpha}\left(\bar{\Omega}^{*}\right)} \leq 2 c_{\kappa} r_{\varepsilon, \lambda}^{2}
$$

As in the previous section, observe that the function $\tilde{v}_{\varepsilon, \tilde{\eta}, \tilde{x}, \tilde{\varphi}, \tilde{\psi}}$ being obtained as a fixed point for contraction mapping depends smoothly on the parameters $\tilde{\eta}^{j}$, the points $\tilde{x}^{j}$, and the boundary data $\tilde{\varphi}^{j}$ and $\tilde{\psi}^{j}$ for $j=1, \ldots, m$. Moreover, as in the previous section, the mapping

$$
\left.(\boldsymbol{\eta}, \tilde{\mathbf{x}}, \tilde{\boldsymbol{\varphi}}, \tilde{\boldsymbol{\psi}}) \mapsto \tilde{v}_{\varepsilon, \boldsymbol{\eta}, \tilde{\mathbf{x}}, \tilde{\varphi}, \tilde{\boldsymbol{\psi}}} \circ D^{-1}\right|_{\Omega_{r_{\varepsilon, \lambda}, \tilde{x}}} \in \mathcal{C}^{4, \alpha}\left(\Omega_{r_{\varepsilon, \lambda}, \tilde{x}}\right)
$$

is compact (here $D$ is the diffeomorphism defined in Sect. 2.2). Again this follows from the fact that the equation we solve is semilinear and in (39) the right-hand side belongs to $\mathcal{C}^{8, \alpha}\left(\bar{\Omega}^{*}\right)$.

\section{The nonlinear Cauchy-data matching}

We will gather the results of the previous sections, keeping the notations, applying the result of Sect. 2, Sect. 3, as well as the results of Sect. 4. Assume that $\tilde{\mathbf{x}}=\left(\tilde{x}^{i}\right) \in \Omega^{m}$ are given close enough to $\mathbf{x}=\left(x^{i}\right)$ such that it satisfies (41), assume also $\boldsymbol{\tau}=\left(\tau^{i}\right) \in\left[\tau^{-}, \tau^{+}\right]^{m} \subset$ $(0, \infty)^{m}$ (the values of $\tau^{-}$and $\tau^{+}$will be fixed shortly). First, we consider some set of boundary data $\varphi=\left(\varphi^{i}\right) \in\left(\mathcal{C}^{4, \alpha}\left(S^{3}\right)\right)^{m}$ and $\boldsymbol{\psi}=\left(\psi^{i}\right) \in\left(\mathcal{C}^{2, \alpha}\left(S^{3}\right)\right)^{m}$ satisfying (26) and (29). According to the result of Proposition 4 and provided $\varepsilon \in\left(0, \varepsilon_{\kappa}\right)$, we can find a solution of

$$
\Delta^{2} u+\mathscr{Q}_{\lambda}(u)-\rho^{4}\left(e^{u}+e^{\gamma u}\right)=0 \quad \text { in } B_{r_{\varepsilon, \lambda}}\left(\tilde{x}^{j}\right) \forall 1 \leq j \leq m .
$$

These solutions can be decomposed (in each $B_{r_{\varepsilon, \lambda}}\left(\tilde{x}^{j}\right)$ ) as

$$
u_{\mathrm{int}, j}(x)=u_{\varepsilon, \tau^{j}}\left(x-\tilde{x}^{j}\right)+h\left(\frac{R_{\varepsilon, \lambda}^{j}\left(x-\tilde{x}^{j}\right)}{r_{\varepsilon, \lambda}}\right)+H_{\varphi^{j}, \psi^{j}}^{i}\left(\frac{x-\tilde{x}^{j}}{r_{\varepsilon, \lambda}}\right)+v_{\varepsilon, \tau^{j}, \varphi^{j}, \psi^{j}}\left(\frac{R_{\varepsilon, \lambda}^{j}\left(x-\tilde{x}^{j}\right)}{r_{\varepsilon, \lambda}}\right),
$$


where $R_{\varepsilon, \lambda}^{j}=\tau^{j} r_{\varepsilon, \lambda} / \varepsilon$ and the function $v^{j}=v_{\varepsilon, \tau^{j}, \varphi^{j}, \psi^{j}}$ satisfies

$$
\left\|v^{j}\right\|_{\mathcal{C}_{\mu}^{4, \alpha}\left(\mathbb{R}^{4}\right)} \leq 2 c_{\kappa} r_{\varepsilon, \lambda}^{2}
$$

Similarly, given some boundary data $\tilde{\boldsymbol{\varphi}}:=\left(\tilde{\varphi}^{i}\right) \in\left(\mathcal{C}^{4, \alpha}\left(S^{3}\right)\right)^{m}$ and $\tilde{\psi}=\left(\tilde{\psi}^{i}\right) \in\left(\mathcal{C}^{2, \alpha}\left(S^{3}\right)\right)^{m}$ satisfying (27) and (40), some parameters $\tilde{\eta}:=\left(\tilde{\eta}^{i}\right) \in \mathbb{R}^{m}$ satisfying (41), we can use the result of Proposition 5 to find a solution $u_{\text {ext }}$ of (provided $\varepsilon \in\left(0, \varepsilon_{\kappa}\right)$ )

$$
\Delta^{2} u+\mathscr{Q}_{\lambda}(u)-\rho^{4}\left(e^{u}+e^{\gamma u}\right)=0, \quad \text { in } B_{r_{\varepsilon, \lambda}}\left(\tilde{x}^{j}\right), \forall 1 \leq j \leq m .
$$

Here the solution can be decomposed as

$$
u_{\mathrm{ext}}(x)=\sum_{j=1}^{m}\left(1+\tilde{\eta}^{j}\right) G_{\tilde{x}^{j}}(x)+\sum_{j=1}^{m} \chi_{r_{0}}\left(x-\tilde{x}^{j}\right) H_{\tilde{\varphi}^{j}, \tilde{\psi}^{j}}^{e}\left(\frac{x-\tilde{x}^{j}}{r_{\varepsilon, \lambda}}\right)+\tilde{v}_{\varepsilon, \tilde{\boldsymbol{\eta}}, \tilde{\mathbf{x}}, \tilde{\boldsymbol{\varphi}}, \tilde{\boldsymbol{\psi}}}(x),
$$

where the function $\tilde{v}^{j}:=\tilde{v}_{\varepsilon, \tilde{\eta}, \tilde{\mathbf{x}}, \tilde{\boldsymbol{\varphi}}, \tilde{\boldsymbol{\psi}}} \in \mathcal{C}_{\nu}^{4, \alpha}\left(\bar{\Omega}^{*}\right)$ satisfies

$$
\left\|\tilde{v}^{j}\right\|_{\mathcal{C}_{v}^{4, \alpha}\left(\bar{\Omega}^{*}\right)} \leq c_{\kappa} r_{\varepsilon, \lambda}^{2}
$$

It remains to determine the parameters and the functions in such a way that the function which is equal to $u_{\text {int, } j}$ in $B_{r_{\varepsilon, \lambda}}\left(\tilde{x}^{j}\right)$ and which is equal to $u_{\text {ext }}$ in $\Omega_{r_{\varepsilon, \lambda}, \tilde{x}}$ will become a smooth function. This amounts to finding the boundary data and the parameters so that, for each $j=1, \ldots, m$,

$$
u_{\mathrm{int}, j}=u_{\mathrm{ext}}, \quad \partial_{r} u_{\mathrm{int}, j}=\partial_{r} u_{\mathrm{ext}}, \quad \Delta u_{\mathrm{int}, j}=\Delta u_{\mathrm{ext}} \quad \text { and } \quad \partial_{r} \Delta u_{\mathrm{int}, j}=\partial_{r} \Delta u_{\mathrm{ext}}
$$

on $\partial B_{r_{\varepsilon, \lambda}}\left(\tilde{x}^{j}\right)$. Assuming we have already (48) (for all $\varepsilon$ small enough), the function $u_{\varepsilon} \in$ $\mathcal{C}^{4, \alpha}$ obtained by patching together the functions $u_{\text {int, } j}$ and the function $u_{\text {ext }}$ is a solution of our equation. Then the elliptic regularity theory implies that this solution is in fact smooth. This will complete the proof of our result. Because as $\varepsilon$ tends to 0 , the sequence of solutions constructed will satisfy the required properties, namely away from the points $x^{j}$ the sequence $u_{\varepsilon}$ converges to $\sum_{j} G_{x}$. Before we proceed, the following remarks are important. It will be convenient to observe that the functions $u_{\varepsilon, \tau^{j}}$ can be expanded as

$$
u_{\varepsilon, \tau^{j}}(x)=-8 \log |x|-4 \log \tau^{j}+\mathcal{O}\left(r_{\varepsilon, \lambda}^{2}\right)
$$

near $\partial B_{r_{\varepsilon, \lambda}}$. Moreover, the function

$$
\sum_{1 \leq j \leq m}\left(1+\tilde{\eta}^{j}\right) G_{\tilde{x}}(x)
$$

which appears in the expression of $u_{\mathrm{ext}}$, can be expanded as

$$
\sum_{\ell=1}^{m}\left(1+\tilde{\eta}^{\ell}\right) G_{\tilde{x}^{\ell}}\left(\tilde{x}^{j}+x\right)=-8\left(1+\tilde{\eta}^{j}\right) \log |x|+E_{j}\left(\tilde{x}^{j}, \tilde{\mathbf{x}}\right)+\nabla_{x} E_{j}\left(\tilde{x}^{j}, \tilde{\mathbf{x}}\right) \cdot x+\mathcal{O}\left(r_{\varepsilon, \lambda}^{2}\right)
$$


near $\partial B_{r_{\varepsilon, \lambda}}$, where we define

$$
E_{j}(x, \tilde{\mathbf{x}}):=H\left(x, \tilde{x}^{j}\right)+\sum_{\ell \neq j} G\left(x, \tilde{x}^{\ell}\right) .
$$

Next, in (48), all functions are defined on $\partial B_{r_{\varepsilon, \lambda}}\left(\tilde{x}^{j}\right)$; nevertheless, it will be convenient to solve, instead of (48), the following set of equations:

$$
\begin{aligned}
& \left(u_{\text {int }, j}-u_{\text {ext }}\right)\left(\tilde{x}^{j}+r_{\varepsilon, \lambda} y\right)=0, \quad \partial_{r}\left(u_{\text {int }, j}-u_{\text {ext }}\right)\left(\tilde{x}^{j}+r_{\varepsilon, \lambda} y\right)=0, \\
& \Delta\left(u_{\text {int }, j}-u_{\text {ext }}\right)\left(\tilde{x}^{j}+r_{\varepsilon, \lambda} y\right)=0, \quad \partial_{r} \Delta\left(u_{\text {int }, j}-u_{\text {ext }}\right)\left(\tilde{x}^{j}+r_{\varepsilon, \lambda} y\right)=0,
\end{aligned}
$$

on $S^{3}$. We decompose also

$$
\varphi^{j}=\varphi_{0}^{j}+\varphi_{1}^{j}+\varphi_{\perp}^{j}, \quad \psi^{j}=8 \varphi_{0}^{j}+12 \varphi_{1}^{j}+\psi_{\perp}^{j}, \quad \tilde{\varphi}^{j}=\tilde{\varphi}_{0}^{j}+\tilde{\varphi}_{1}^{j}+\tilde{\varphi}_{\perp}^{j} \quad \text { and } \quad \tilde{\psi}^{j}=\tilde{\psi}_{1}^{j}+\tilde{\psi}_{\perp}^{j},
$$

where $\varphi_{0}^{j}, \tilde{\varphi}_{0} \in \mathbb{E}_{0}=\mathbb{R}, \varphi_{1}^{j}, \tilde{\varphi}_{1}^{j}, \tilde{\psi}_{1}^{j} \in \mathbb{E}_{1}=\operatorname{Span}\left\{e_{1}, \ldots, e_{4}\right\}$, and $\varphi_{\perp}^{j}, \psi_{\perp}^{j}, \tilde{\varphi}_{\perp}^{j}, \tilde{\psi}_{\perp}^{j} \in L^{2}\left(S^{3}\right)^{\perp}$, the subspace of functions which are orthogonal to $\mathbb{E}_{0}$ and $\mathbb{E}_{1}$. Projecting the set of equations (51) over $\mathbb{E}_{0}$ will yield the system

$$
\left\{\begin{array}{l}
-4 \log \tau^{j}-8 \log r_{\varepsilon, \lambda}+\varphi_{0}^{j}+8\left(1+\tilde{\eta}^{j}\right) \log r_{\varepsilon, \lambda}-\tilde{\varphi}_{0}^{j}-E_{j}\left(\tilde{x}^{j}, \tilde{\mathbf{x}}\right)+\mathcal{O}\left(r_{\varepsilon, \lambda}^{2}\right)=0, \\
-8+2 \varphi_{0}^{j}+8\left(1+\tilde{\eta}^{j}\right)+2 \tilde{\varphi}_{0}^{j}+\mathcal{O}\left(r_{\varepsilon, \lambda}^{2}\right)=0, \\
-16+8 \varphi_{0}^{j}+16\left(1+\tilde{\eta}^{j}\right)+\mathcal{O}\left(r_{\varepsilon, \lambda}^{2}\right)=0, \\
32-32\left(1+\tilde{\eta}^{j}\right)+\mathcal{O}\left(r_{\varepsilon, \lambda}^{2}\right)=0 .
\end{array}\right.
$$

Here, and from now on, the term $\mathcal{O}\left(r_{\varepsilon, \lambda}^{2}\right)$ depends nonlinearly on the variables $\tau^{\ell}, \tilde{x}^{\ell}, \varphi^{\ell}, \psi^{\ell}$, $\tilde{\varphi}^{\ell}, \tilde{\psi}^{\ell}$, but it is bounded (in the appropriate norm) by a constant (independent of $\varepsilon$ and $\kappa$ ) time $r_{\varepsilon, \lambda}^{2}$. Let us comment briefly on how these equations are obtained. These equations simply come from (51) when expansions (49) and (50) are used, together with the expressions of $H^{i}$ and $H^{e}$ given in Lemma 2 and Lemma 3, also estimates (46) and (47). Observe that the projection of the term $\nabla_{x} E_{j}\left(\tilde{x}^{j}, \tilde{\mathbf{x}}\right) \cdot y$ arising in (50), as well as the projection of its partial derivative with respect to $r$, over the set of constant functions is equal to 0 , while its Laplacian vanishes identically. System (52) can be readily simplified into

$$
\begin{aligned}
& \frac{1}{\log r_{\varepsilon, \lambda}}\left[4 \log \tau^{j}+E_{j}\left(\tilde{x}^{j}, \tilde{\mathbf{x}}\right)\right]=\mathcal{O}\left(r_{\varepsilon, \lambda}^{2}\right), \quad \tilde{\eta}^{j}=\mathcal{O}\left(r_{\varepsilon, \lambda}^{2}\right), \\
& \varphi_{0}^{j}=\mathcal{O}\left(r_{\varepsilon, \lambda}^{2}\right) \quad \text { and } \quad \tilde{\varphi}_{0}^{j}=\mathcal{O}\left(r_{\varepsilon, \lambda}^{2}\right) .
\end{aligned}
$$

We are now in a position to define $\tau^{-}$and $\tau^{+}$since, according to the above, as $\varepsilon$ tends to 0 , we expect that $\tilde{x}^{j}$ will converge to $x^{j}$ and that $\tau^{j}$ will converge to $\tau_{*}^{j}$ satisfying

$$
4 \log \tau_{*}^{j}=-E_{j}\left(x^{j}, \mathbf{x}\right)
$$

and hence it is enough to choose $\tau^{-}$and $\tau^{+}$in such a way that

$$
4 \log \left(\tau^{-}\right)<-\sup _{j} E_{j}\left(x^{j}, \mathbf{x}\right) \leq-\inf _{j} E_{j}\left(x^{j}, \mathbf{x}\right)<4 \log \left(\tau^{+}\right) .
$$


We now consider the $L^{2}$-projection of (51) over $\mathbb{E}_{1}$. Given a smooth function $f$ defined in $\Omega$, we identify its gradient $\nabla f=\left(\partial_{x_{1}} f, \ldots, \partial_{x_{4}} f\right)$ with the element of $\mathbb{E}_{1}$

$$
\bar{\nabla} f=\sum_{i=1}^{4} \partial_{x_{i}} f e_{i} .
$$

With these notations in mind, we obtain the system of equations

$$
\left\{\begin{array}{l}
\varphi_{1}^{j}-\tilde{\varphi}_{1}^{j}-\bar{\nabla} E_{j}\left(\tilde{x}^{j}, \tilde{\mathbf{x}}\right)+\mathcal{O}\left(r_{\varepsilon, \lambda}^{2}\right)=0, \\
3 \varphi_{1}^{j}+3 \tilde{\varphi}_{1}^{j}+\frac{1}{2} \tilde{\psi}_{1}^{j}-\bar{\nabla} E_{j}\left(\tilde{x}^{j}, \tilde{\mathbf{x}}\right)+\mathcal{O}\left(r_{\varepsilon, \lambda}^{2}\right)=0, \\
12 \varphi_{1}^{j}-\tilde{\varphi}_{1}^{j}+\mathcal{O}\left(r_{\varepsilon, \lambda}^{2}\right)=0, \\
12 \varphi_{1}^{j}+3 \tilde{\varphi}_{1}^{j}+\mathcal{O}\left(r_{\varepsilon, \lambda}^{2}\right)=0 .
\end{array}\right.
$$

Again, let us comment briefly on how these equations are obtained. This time, the only important observation is that the term $\nabla_{x} E_{j}\left(\tilde{x}^{j}, \tilde{\mathbf{x}}\right) \cdot y$ projects identically over $\mathbb{E}_{1}$ as well as its derivative with respect to $r$. System (53) simplifies into

$$
\varphi_{1}^{j}=\mathcal{O}\left(r_{\varepsilon, \lambda}^{2}\right), \quad \psi_{1}^{j}=\mathcal{O}\left(r_{\varepsilon, \lambda}^{2}\right), \quad \tilde{\psi}_{1}^{j}=\mathcal{O}\left(r_{\varepsilon, \lambda}^{2}\right) \quad \text { and } \quad \bar{\nabla} E_{j}\left(\tilde{x}^{j}, \tilde{\mathbf{x}}\right)=\mathcal{O}\left(r_{\varepsilon, \lambda}^{2}\right) .
$$

Finally, we consider the $L^{2}$-projection onto $L^{2}\left(S^{3}\right)^{\perp}$. This yields the system

$$
\left\{\begin{array}{l}
\varphi_{\perp}^{j}-\tilde{\varphi}_{\perp}^{j}+\mathcal{O}\left(r_{\varepsilon, \lambda}^{2}\right)=0, \\
\partial_{r}\left(H_{\varphi_{\perp}, \psi_{\perp}^{j}}^{i}-H_{\tilde{\varphi}_{\perp}^{j}, \tilde{\psi}_{\perp}^{j}}^{e}\right)+\mathcal{O}\left(r_{\varepsilon, \lambda}^{2}\right)=0, \\
\psi_{\perp}^{j}-\tilde{\psi}_{\perp}^{j}+\mathcal{O}\left(r_{\varepsilon, \lambda}^{2}\right)=0, \\
\partial_{r} \Delta\left(H_{\varphi_{\perp}, \psi_{\perp}^{j}}^{i}-H_{\tilde{\varphi}_{\perp}, \tilde{\psi}_{\perp}^{j}}^{e}\right)+\mathcal{O}\left(r_{\varepsilon, \lambda}^{2}\right)=0 .
\end{array}\right.
$$

Thanks to the result of Lemma 4, this last system can be re-written as

$$
\varphi_{\perp}^{j}=\mathcal{O}\left(r_{\varepsilon, \lambda}^{2}\right) \quad \text { and } \quad \psi_{\perp}^{j}=\mathcal{O}\left(r_{\varepsilon, \lambda}^{2}\right)
$$

If we define the parameters $\mathbf{t}=\left(t^{j}\right) \in \mathbb{R}^{m}$ by

$$
t^{j}=\frac{1}{\log r_{\varepsilon, \lambda}}\left[4 \log \tau^{j}+E_{j}\left(\tilde{x}^{j}, \tilde{x}\right)\right], \quad \forall 1 \leq j \leq m,
$$

then the system we have to solve reads

$$
\left(\mathbf{t}, \tilde{\boldsymbol{\eta}}, \boldsymbol{\varphi}_{0}, \tilde{\boldsymbol{\varphi}}_{0}, \boldsymbol{\varphi}_{1}, \tilde{\boldsymbol{\varphi}}_{1}, \tilde{\boldsymbol{\psi}}_{1}, \bar{\nabla} E(\tilde{\mathbf{x}}), \boldsymbol{\varphi}_{\perp}, \tilde{\boldsymbol{\varphi}}_{\perp}, \boldsymbol{\psi}_{\perp}, \tilde{\psi}_{\perp}\right)=\mathcal{O}\left(r_{\varepsilon, \lambda}^{2}\right)
$$

where, as usual, the term $\mathcal{O}\left(r_{\varepsilon, \lambda}^{2}\right)$ depends nonlinearly on all the variables on the left side, but is bounded (in the appropriate norm) by a constant (independent of $\varepsilon$ and $\kappa$ ) time $r_{\varepsilon, \lambda}^{2}$, provided $\varepsilon \in\left(0, \varepsilon_{\kappa}\right)$. We claim that, provided that the degree of the mapping

$$
\bar{\nabla} E: \tilde{\mathbf{x}} \longmapsto\left(\bar{\nabla} E_{1}\left(\tilde{x}^{1} ; \tilde{\mathbf{x}}\right), \ldots, \bar{\nabla} E_{m}\left(\tilde{x}^{m} ; \tilde{\mathbf{x}}\right)\right)
$$


from a neighborhood of $\mathbf{x} \in \Omega^{m}$ to a neighborhood of 0 in $\mathbb{E}_{1}^{m}$ is equal to 1 , this nonlinear system can be solved using Schauder's fixed point theorem in the ball of radius $\kappa r_{\varepsilon, \lambda}^{2}$ in the product space where the entries live, namely

$$
\mathbf{t}, \boldsymbol{\eta} \in \mathbb{R}^{m} ; \quad r_{\varepsilon, \lambda}(\tilde{\boldsymbol{x}}-\mathbf{x}) \in\left(\mathbb{R}^{4}\right)^{m} ; \quad \boldsymbol{\varphi}_{0}, \tilde{\boldsymbol{\varphi}}_{0} \in \mathbb{R}^{m}
$$

and

$$
\boldsymbol{\varphi}_{1}, \tilde{\boldsymbol{\varphi}}_{1}, \tilde{\boldsymbol{\psi}}_{1} \in \mathbb{E}_{1}^{m} ; \quad \boldsymbol{\varphi}_{\perp}, \tilde{\boldsymbol{\varphi}_{\perp}}, \boldsymbol{\boldsymbol { \psi } _ { \perp }}, \tilde{\boldsymbol{\varphi}_{\perp}} \in\left(\mathcal{C}^{2, \alpha}\left(S^{3}\right)^{\perp}\right)^{m}
$$

Indeed, the nonlinear mapping which appears on the right-hand side of (55) is continuous, compact. In addition, this nonlinear mapping sends the ball of radius $\kappa r_{\varepsilon, \lambda}^{2}$ (for the natural product norm) into itself, provided $\kappa$ is fixed large enough. In order to obtain the precise statement of our theorem, we simply observe that

$$
2 \nabla_{x} E_{j}(\tilde{x}, \tilde{\mathbf{x}})=\nabla_{\tilde{x}^{j}} E(\tilde{\mathbf{x}})
$$

where $E$ is the functional defined by (12), then a sufficient condition for mapping (56) to have degree 1 is just that the point $\mathbf{x}=\left(x^{1}, \ldots, x^{m}\right)$ is a nondegenerate critical point of the functional $E$. This completes the proof of our theorem.

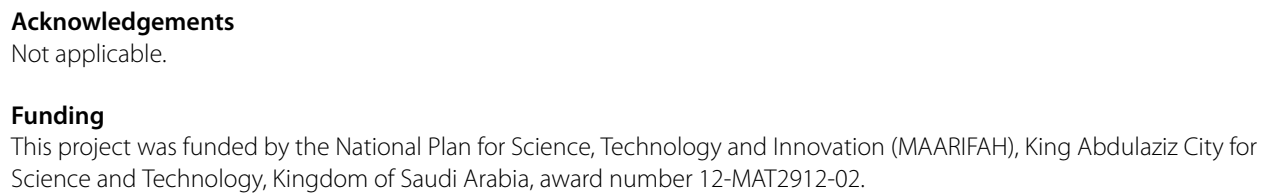

Abbreviations

Not applicable.

Availability of data and materials

Not applicable.

Competing interests

The authors declare that there are no competing interests.

Authors' contributions

The authors completed the paper and approved the final manuscript.

\section{Author details}

'Department of Mathematics, Faculté des Sciences de Tunis, University Tunis El Manar, Tunis, Tunisie. ${ }^{2}$ Department of Mathematics, College of Science, King Saud University, Riyadh, Saudi Arabia.

\section{Publisher's Note}

Springer Nature remains neutral with regard to jurisdictional claims in published maps and institutional affiliations.

Received: 28 May 2019 Accepted: 23 July 2019 Published online: 01 August 2019

\section{References}

1. Arioli, G., Gazzola, F., Grunau, H.C., Mitidieri, E.: A semilinear fourth order elliptic problem with exponential nonlinearity. SIAM J. Math. Anal. 36(4), 1226-1258 (2005)

2. Baraket, S., Bazarbacha, I., Trabelsi, M.: Singular limiting solutions to 4-dimensional elliptic problems involving exponentially dominated nonlinearity and nonlinear terms. Electron. J. Differ. Equ. 2015, 289 (2015)

3. Baraket, S., Bazarbacha, I., Trabelsi, N.: Construction of singular limits for 4-dimensional elliptic problems with exponentially dominated nonlinearity. Bull. Sci. Math. 31, 670-685 (2007)

4. Baraket, S., Dammak, M., Ouni, T., Pacard, F.: Singular limits for a 4-dimensional semilinear elliptic problem with exponential nonlinearity. Ann. Inst. Henri Poincaré, Anal. Non Linéaire 24, 875-895 (2007) 
5. Baraket, S., Pacard, F.: Construction of singular limits for a semilinear elliptic equation in dimension. Calc. Var. Partial Differ. Equ. 6, 1-38 (1998)

6. Ben Ayed, M., El Mehdi, K., Grossi, M.: Asymptotic behavior of least energy solutions of biharmonic equation in dimension four. Indiana Univ. Math. J. 5, 1723-1750 (2006)

7. Branson, T.: Group representations arising from Lorentz conformal geometry. J. Funct. Anal. 74, 199-293 (1987)

8. Branson, T.: Sharp inequality, the functional determinant and the complementary series. Trans. Am. Math. Soc. 347, 3671-3742 (1995)

9. Chang, S.Y.A.: On a fourth order differential operator-the Paneitz operator in conformal geometry, proceedings conference for the 70th birthday of A.P. Calderon 4, 155-165 (2000)

10. Chang, S.Y.A., Yang, P.: On a fourth order curvature invariant. In: Branson, T. (ed.) Contemporary Mathematics. AMS, and Arithmetic, vol. 237, pp. 9-28. AMS, Providence (1999)

11. Clapp, M., Munoz, C., Musso, M.: Singular limits for the bi-Laplacian operator with exponential nonlinearity in $\mathbb{R}^{4}$. Ann Inst. Henri Poincaré 25, 1015-1041 (2008)

12. Del Pino, M., Kowalczyk, M., Musso, M.: Singular limits in Liouville type equations. Calc. Var. Partial Differ. Equ. 24(1), 47-81 (2005)

13. Esposito, P., Grossi, M., Pistoia, A.: On the existence of blowing-up solutions for a mean field equation. Ann. Inst. Henri Poincaré 22, 227-257 (2005)

14. Liouville, J.: Sur l'équation aux différences partielles $\partial^{2} \log \frac{\lambda}{\partial u \partial v} \pm \frac{\lambda}{2 a^{2}}=0$. J. Math. 18, $17-72$ (1853)

15. Malchiodi, A., Djadli, Z.: Existence of conformal metrics with constant Q-curvature. Ann. Math. 168(3), 813-858 (2008)

16. Mazzeo, R.: Elliptic theory of edge operators I. Commun. Partial Differ. Equ. 10(16), 1616-1664 (1991)

17. Melrose, R.: The Atiyah-Patodi-Singer Index Theorem. Res. Notes Math., vol. 4 (1993)

18. Pacard, F., Rivière, T.: Linear and nonlinear aspects of vortices: the Ginzburg Landau model. In: Progress in Nonlinear Differential Equations, vol. 39. Birkäuser, Basel (2000)

19. Suzuki, T.: Two dimensional Emden-Fowler equation with exponential nonlinearity. In: Nonlinear Diffusion Equations and Their Equilibrium States 3, pp. 493-512. Birkäuser, Basel (1992)

20. Wei, J.: Asymptotic behavior of a nonlinear fourth order eigenvalue problem. Commun. Partial Differ. Equ. 21(9-10), 1451-1467 (1996)

21. Wente, H.C.: Counter example to a conjecture of H. Hopf. Pac. J. Math. 121, 193-243 (1986)

\section{Submit your manuscript to a SpringerOpen ${ }^{\circ}$ journal and benefit from:}

- Convenient online submission

- Rigorous peer review

- Open access: articles freely available online

- High visibility within the field

Retaining the copyright to your article

Submit your next manuscript at $\gg$ springeropen.com 